\title{
Klingeltöne und Logos auf dem Handy: Wie neue Medien der Uni-Kommunikation genutzt werden*
}

\author{
Nicola Döring
}

Indem Handybesitzer gegenüber anderen Menschen in systematischer Weise ibr Endgerät verbergen oder zur Schau stellen und indem sie es durch die Auswabl bestimmter Klingeltöne und Logos für Außenstehende wabrnehmbar mit zusätzlicher Symbolik ausstatten, können sie dem jeweiligen Publikum etwas über ibren Status, ibre Einstellungen, ibre Interessen und Gruppenzugebörigkeiten mitteilen (Uni-Kommunikation). Dervorliegende Beitrag rekonstruiert die Nutzung von Klingeltönen und Logos auf dem Handy als uni-kommunikatives Geschehen und liefert Daten aus drei explorativen Studien: 1. einer quantitativen Inhaltsanalyse von Klingelton-und Logo-Angeboten im Internet, 2. einer leitfadengestützten mündlichen Befragung sowie 3. einer vollstrukturierten Online-Befragung von Handy-Nutzern. Es zeigte sich, dass die Häufigkeit, mit der neue Klingeltöne und Logos auf das Handy geladen werden, sehr stark variiert. Die Themenwabl (z. B. Klingelton mit Pop-, Rock-oder Klassik-Melodie; Logo mit Tier-, Liebes-, Sex-, TV-oder Auto-Motiv) korreliert eng mit individuellen Interessen, Geschlecht und Alter. Im sozialen Kontext werden Klingeltöne und Logos tatsächlich zum Gegenstand der Aufmerksamkeit und Eindrucksbildung und stimulieren teilweise interpersonale sowie intra- und intergruppale Anschluss-Kommunikation.

Keywords: Mobilkommunikation, Uni-Kommunikation, Handy, Logos, Klingeltöne, Mediennutzung

\section{O. Einleitung}

Im Jahr 2002 wurden weltweit erstmals mehr Mobiltelefone als Festnetzanschlüsse registriert (International Telecommunication Union, 2002). Zudem übertrifft die Zahl der Mobiltelefone mittlerweile die der Fernsehgeräte (Katz \& Aakhus, 2002b: 4). Sowohl in privaten als auch in (teil-)öffentlichen Situationen sind Handys heute allgegenwärtig. Mobiltelefone machen mit den unterschiedlichsten Klingeltönen (ringing tones/ring tones) auf eingehende Anrufe aufmerksam - und zwar nicht nur den Handybesitzer, sondern auch andere Ohrenzeugen. Man kann zwischen einigen auf dem Handy serienmäßig mitgelieferten Melodien wählen oder sich via Internet bzw. Service-Rufnummer neue Töne auf das Handy laden, sofern das jeweilige Endgerät dies zulässt. Auch Eigenkompositionen sind möglich. Dasselbe gilt für selbst gewählte Logos (operator logos), die an Stelle des voreingestellten Netzbetreiberlogos auf dem Handy-Display angezeigt werden. Wenn das Handy auf dem Tisch liegt oder zur Hand genommen wird, ist das Handylogo nicht nur für den Besitzer, sondern auch für weitere Augenzeugen

* Ich danke Katja Andreä, Christine Dietmar und Carolin Kühndelt (Studiengang Angewandte Medienwissenschaft, Technische Universität Ilmenau) für ihre tatkräftige Unterstützung bei der Datenerhebung und Datenauswertung. 
sichtbar. Zahlreiche Logo-Motive stehen zum Herunterladen zur Verfügung, ebenso existieren Editoren für Eigenkreationen. Handylogos erlauben genau wie Klingeltöne eine individualisierte Gestaltung und bessere Wiedererkennbarkeit des eigenen Endgerätes. Zudem sind sie aber auch Träger kommunikativer Botschaften. So kommentiert Joachim Höflich (2001: 4): Der Rufton des Handys - „der bis hin zu äußerst lauten und manchmal nie enden wollenden Melodien reicht, von Pop bis Klassik (was immer die Handybesitzer damit sagen wollen) - lässt Dritte nicht unberührt“.

Eben jene kommunikativen Aspekte der Nutzung von Klingeltönen und Logos sollen hier untersucht werden. Denn während viele Handybesitzerinnen und -besitzer Klingeltöne und Logos als teure und sinnlose Spielereien betrachten und dementsprechend nicht nutzen, greifen andere in so großer Zahl bzw. so regelmäßig auf sie zurück, dass der kostenpflichtige Klingelton- und Logo-Vertrieb per Internet oder Service-Rufnummer sich seit 1998 als neues Geschäftsfeld etablieren konnte und mit Anzeigenkampagnen vor allem in Jugendzeitschriften stark beworben wird. Neben der SMS-Kommunikation (vgl. Höflich \& Rössler, 2001; Döring, 2002) und der Mobiltelefonie sind speziell bei europäischen Jugendlichen Klingeltöne und Logos sehr populär. Gut fünf Euro gaben norwegische Handynutzer aller Altersgruppen im Jahr 2000 durchschnittlich allein für Klingeltöne aus (Strand Consult, 2001). Trotz verhältnismäßig kleiner Einzelbeträge wurde auf dem Europäischen Markt für mobilen Content (Klingeltöne, Logos, SMS-Informationsdienste, Bildmitteilungen usw.) durch die große Zahl der Nutzer bereits 2001 mehr als doppelt so viel Geld umgesetzt (590 Millionen US-Dollar) wie auf dem Markt für Online-Content (252 Millionen US-Dollar, Jupiter MMXI, 2002). Die Besonderheit von Klingeltönen und Logos als mobilem Content besteht darin, dass sie nicht einfach einmalig vom Nutzer rezipiert, sondern über das mobile Endgerät permanent in soziale und (teil-)öffentliche Kommunikationsprozesse integriert werden.

Der vorliegende Beitrag rekonstruiert zunächst das Handy mit seinen Klingeltönen und Logos als neues Medium der Uni-Kommunikation und formuliert fünf Forschungsfragen (Kap. 1). Auf die Beschreibung der Untersuchungsmethoden (Kap. 2) folgt die Darstellung der explorativen Ergebnisse zu Nutzungshäufigkeiten, Themenwahlen und sozialen Konsequenzen (Kap. 3). Der Aufsatz endet mit einer kurzen Diskussion (Kap. 4).

\section{Gegenstand und Fragestellungen}

Das Handy ist nicht nur ein Medium der Telekommunikation, sondern lässt sich auch als Medium der Uni-Kommunikation verstehen, wobei kommunikative Aussagen theoretisch vor allem über Klingeltöne und Logos vermittelt werden können. Anhand des Konstrukts der Uni-Kommunikation werden erste Forschungsfragen entwickelt, um zu erkunden, ob und wie Klingeltöne und Logos tatsächlich als neue Medien der Uni-Kommunikation genutzt werden.

\subsection{Uni-Kommunikation per Handy}

„Uni-communication is that communication mediated by objects of clothing, adornment, and personal possessions - houses, automobiles, furniture, etc. which people select and display to communicate to others their status, affiliation, and self-esteem. It includes, also, more explicit messages like imprinted T-shirts, jackets, and caps, as well as bumperstickers, armbands, and buttons.“ (Cathcart \& Gumpert, 1983: 275f.). 
Gary Gumpert (Professor Emeritus of Communication Arts and Sciences, City University of New York) führte das Konstrukt der „Uni-Kommunikation“ erstmals 1975 ein. Er wollte darauf aufmerksam machen, dass mediatisierte Kommunikation nicht nur von Medienorganisationen ausgeht, sondern auch Gruppen und Individuen als Kommunikatoren aktiv werden und unterschiedliche Publika adressieren. Er ergänzte den eingeführten Begriff der „Massen-Kommunikation“ um die Wortneuschöpfungen „Mini-Kommunikation“ (Gumpert, 1970) und „Uni-Kommunikation“ (Gumpert, 1975; Cathcart \& Gumpert, 1983: 275f.; Gumpert \& Drucker, 1999: 17). „Uni“ bezieht sich hierbei auf die Einzelperson, die Alltagsgegenstände und den eigenen Körper (z. B. Tattoo, Piercing) nutzt, um selbstbezogene Botschaften an unterschiedliche Publika zu richten (persönliche E-Mail-Mitteilung von Gary Gumpert vom 26. Juni 2002). UniKommunikation ist eine Form der mediatisierten Kommunikation, die in Face-to-FaceSituationen auftritt, ohne dass die Beteiligten direkt miteinander sprechen müssen, können oder wollen. Wer sich mit einem „Atomkraft: Nein Danke“-Aufkleber auf dem Auto oder einem „Playboy“-Aufdruck auf dem T-Shirt in der Öffentlichkeit bewegt, macht damit in der jeweiligen Situation eine oder mehrere selbstbezogene Aussagen, die wiederum von denjenigen, die diese medialen Botschaften wahrnehmen, interpretiert und mit entsprechenden inneren und äußeren Reaktionen beantwortet werden - zuweilen bis hin zu Zeitungs-Kommentaren (Zykla, 2001). Durch Uni-Kommunikation kann das Individuum Aspekte der eigenen Identität für sich selbst bekräftigen und vor anderen ein bestimmtes Image aufbauen.

Das körpernah getragene und in diversen öffentlichen Situationen genutzte Mobiltelefon, das im Unterschied zum Festnetztelefon als persönliches Mehrzweckmedium angeeignet wird (Höflich, 2001), dient neben der Telekommunikation verstärkt auch der Uni-Kommunikation.

Als Objekt ist das Handy überwiegend affektiv positiv aufgeladen und lässt soziale Verbindung, Sicherheit, Offenheit gegenüber der Welt und Vergnügen assoziieren (Fortunati, 1997). Wer per Handy häufig Freundschafts- und Liebesgrüße, lustige Sprüche und romantische Gedichte oder eben Klingeltöne und Logos austauscht, erlebt das Gerät sogar als „Geschenk-Container“ (Harper, 2002: 221f.), dessen Präsentation das Selbstwertgefühl steigert. Andererseits wird Handybesitz bei Jugendlichen manchmal auch mit Kriminalität (vor allem Drogenhandel) in Verbindung gebracht, was dann das eigene öffentliche Image gefährdet (Robbins \& Turner, 2002: 90).

Sadie Plant (2001) konnte mittels Feldbeobachtungen in Restaurants und Bars zeigen, dass Personen ihre Handys in systematischer Weise zur Schau stellen oder verbergen. Der Besitz eines Handys - speziell eines besonders teuren oder ausgefallenen Modells signalisiert Beobachtern den Status, die jeweils eingenommene Rolle oder den Lebensstil des Besitzers. „Handys sind die einzigen Objekte, bei denen Männer sich streiten, wer das kleinere hat" - dieser im World Wide Web tausendfach verbreitete Spruch verweist auf die uni-kommunikative Funktion des Handys im Bereich der Geschlechterkonstruktionen. Tatsächlich bestätigten die in englischen Bars durchgeführten Feldstudien die Mutmaßung, dass Menschen mit der Präsentation ihres Handys Geschlechtsidentitäten inszenieren:

„In the majority of pairs of men, at least one mobile was on display: in only 38 per cent of observed pairs was there no mobile on display, compared to 42 per cent of male and female pairs, and 50 per cent of female pairs. In the majority of observed male pairs, both parties had their mobiles on show. Some contributors to the research suggested that this reflected a degree of competitive be- 
haviour among men, with the presence of just one mobile indicating a subtle play of dominance and subordination in which the male who displays his mobile is also asserting his position as the pair's main contact with the wider world. A number of males also confessed to being inhibited when their companions displayed mobiles of a higher specification or aesthetic quality than their own. Others said they had been keen to display their mobiles while they were topof-the-range or state-of-the-art, but had stopped doing so when their models fell behind." (Plant, 2001: 12)

John Lycett und Robin Dunbar (2000) interpretierten den öffentlich beobachtbaren Umgang von Männern mit ihren Handys in einer Bar als „Balzverhalten“ und Anthony Townsend (2002: 67f.) weist auf eine deutliche Geschlechtsrollenorientierung in der Handy-Werbung hin: Für die weibliche Zielgruppe wurde das Handy bislang häufig mit Sicherheit und sozialer Vernetzung assoziiert, für die männliche mit Macht und Virilität. Um das uni-kommunikative Potenzial des Mobiltelefons und seiner Applikationen für die Konstruktion von Geschlechteridentitäten zu untersuchen, sind freilich auch die Reaktionen des Publikums auf entsprechende mediengestützte Selbstdarstellungen einzubeziehen. So kann anekdotisch das Handy mit einem konkreten Klingelton in Kombination mit dem passenden Auftreten der Handy-Besitzerin offensichtlich auch ein bestimmtes Bild von Weiblichkeit unterstreichen (s. u. 3.5).

\subsection{Klingeltöne und Logos auf dem Handy}

Noch expliziter als Medium der Uni-Kommunikation kann das Handy immer dann fungieren, wenn es von der Nutzerin oder dem Nutzer bewusst als Symbolträger verwendet wird, wobei Klingeltöne (Ruftöne) und Betreiberlogos die gängigsten Formate sind. Sie werden Software-seitig über die SIM-Karte (Subscriber Identification Module) realisiert. Die SIM-Karte ist eine individuelle Speicherkarte, die in das Handy eingesetzt wird und es betriebsfähig macht. Mit Klingeltönen verwandte auditive Produkte sind vorformulierte Anrufbeantworter-Ansagen (Mailbox/Mobilbox-Sprüche). Mit Betreiberlogos verwandte grafische Produkte sind Anrufergruppen-Symbole (Gruppenlogos), die aktuell eingehende Anrufe bestimmter Personen bzw. Personengruppen signalisieren und somit nur temporär erscheinen. Sie sind für Außenstehende meist nicht so deutlich erkennbar wie Betreiberlogos, die im Stand-By-Modus permanent angezeigt werden. Daneben lassen sich kommunikative Botschaften auch Hardware-seitig am Handy anbringen, sei es durch Handy-Taschen oder Handy-Halter mit entsprechenden Aufschriften, durch Aufkleber, Anhänger, Lackierungen oder auswechselbare Oberschalen. Die Hardware-seitigen Varianten der Handy-Gestaltung sind weniger verbreitet als die Software-seitigen und werden hier nicht betrachtet. Insgesamt bleibt festzuhalten, dass Hardware- und Software-seitiges Handy Tuning als uni-kommunikative Strategie an Bedeutung gewinnt, je weniger distinktiv Handy-Besitz oder Handy-Marke sind.

Eine kleine Auswahl an serienmäßigen Klingeltönen und Logos bzw. Anrufergruppen-Symbolen wird bei vielen Handytypen heute mitgeliefert und kann vom Nutzer wahlweise aktiviert werden. Wer sich zusätzliche Klingeltöne oder Betreiberlogos auf sein Handy laden will, nutzt beispielsweise eine Website, wobei es sich etwa um die Online-Präsenzen von Netz- und Service-Providern (z. B. www.t-mobile.de), um die Homepages von klassischen Massenmedien (z. B. www.bravo.de; www.bild.de; www.mtv.de), um dezidierte Handy-Portale (z. B. www.handy.de; www.jamba.de) oder auch um spezialisierte Download-Sites handeln kann (z. B. www.handylogos.de; 
www.klingeltoene.de; www.klingeltoene-handylogo.de). Auf den Websites werden Klingeltöne und Betreiberlogos nach inhaltlichen Rubriken sortiert dargestellt. Der Versand der ausgewählten Klingeltöne und Betreiberlogos erfolgt per SMS in einem vom Handyhersteller abhängigen Format und wird über unterschiedliche Bezahlmodelle abgewickelt:

- Bei einer Internet-Bestellung werden Klingeltöne und Logos auf einer Website ausgesucht. Dann ist über eine kostenpflichtige Service-Rufnummer eine einmal gültige PIN (Personal Identification Number) bzw. ein Jeton abzurufen (6-stellige Ziffer) und zusammen mit der Telefonnummer des Zielhandys in das Online-Bestellformular einzutragen.

- Bei einer Telefon-Bestellung ist wiederum eine Service-Rufnummer (0190er Nummer) anzurufen, wobei Handynummer sowie Bestellnummer für den ausgewählten Klingelton bzw. das ausgewählte Logo telefonisch durchgegeben werden. Die Auswahl der Klingeltöne und Logos erfolgt dabei anhand von Anzeigen in Printmedien (typischerweise in Jugendzeitschriften wie „Bravo“ oder „Sugar“ oder in Programmzeitschriften wie „TV Movie“), denen Bestellnummern und Service-Rufnummern zu entnehmen sind.

- Bei der Bestellung mittels Mobiltelefon gibt es zwei Varianten: zum einen die SMSBestellung, bei der die Bestellnummer des Klingeltons bzw. Logos per SMS an eine bestimmte Telefonnummer geschickt wird, und zum anderen die WAP-Bestellung (WAP: Wireless Application Protocol), bei der auf ein mobiles Internet-Portal zugegriffen wird (z. B. wap.jamba.de).

Die Bestell-Kosten pro Klingelton bzw. Logo variieren je nach Anbieter, Minutenpreis der 0190er-Rufnummer (z. B. 1,86 Euro/Min.) und Dauer des Bestellvorgangs (z. B. 45 Sekunden), wobei ein Download-Preis von ca. 1,5 Euro pro Klingelton oder Logo typisch ist. Einige Websites bieten in begrenztem Umfang kostenlose Downloads an (z. B. www.nur-gratis-ringtones.de; www.klingeltonwelt.com). Es besteht die Möglichkeit, bereits heruntergeladene Klingeltöne und Logos per SMS direkt mit anderen HandyNutzern auszutauschen, wobei dann SMS-Gebühren anfallen. Zudem erlauben es entsprechende Editoren (z. B. Logo-Manager, Logo-Composer, Ring-Master), die mit dem Handy auf einem Datenträger ausgeliefert werden oder per Internet zu beziehen sind, Klingeltöne und Logos am Computer selbst zu erstellen und per Datenkabel oder Infrarot-Schnittstelle auf das eigene Handy zu übertragen. Manche Handytypen enthalten auch bereits einen integrierten Klingelton-Composer. Schließlich lassen sich vorhandene Audio- und Grafikdateien (z. B. im WAV-, MIDI- oder BMP-Format) als Klingeltöne und Logos nutzen. Die ersten Klingelton- und Logo-fähigen Handys stammten vom Marktführer Nokia, entsprechend verbreitet sind die Nokia-Formate RTTTL (Ringing Tone Text Transfer Language) und NOL (Nokia Operator Logo); es existieren jedoch auch andere Formate sowie Konvertierungstools.

Anbieter beziehen Klingeltöne und Logos von Mobile-Content-Lieferanten und/ oder produzieren ihren Content selbst. Phat Tonez (www.tonez.co.uk), Wireless Entertainment Services Finland Ltd. (www.wirelessfun.com) und DAC-Planet GmbH (www.dac-planet.de) - alle im Jahr 1999 gegründet - sind Beispiele für international tätige Mobile-Content-Lieferanten mit Schwerpunkt Klingeltöne und Logos. Ihre Produktion von Klingeltönen orientiert sich unter anderem an nationalen Pop-Charts und beinhaltet auch die Kooperation mit einzelnen Künstlern (z. B. Madonna). Die Qualität der Klingeltöne bemisst sich an dem gewählten Melodieausschnitt sowie an der Sorgfalt der Nachkomposition. Für Musiktitel, die in Form von Klingeltönen auf Handys weitergegeben werden, sind 15 Prozent vom Endkundenpreis als Vergütung an die Musik- 
rechte-Verwertungsgesellschaft GEMA zu zahlen (GEMA, 2001). Für den Entwurf von Logos werden Grafiker beschäftigt, wobei der Rückgriff auf bekannte Cartoon-Figuren wiederum urheber- und lizenzrechtlich abgesichert werden muss. In den aktuellen Mobilfunknetzen der 2. Generation (GSM: Global System for Mobile Communications) sind die mit herkömmlichen Handys genutzten Klingeltöne typischerweise monophon und die Logos monochrom. Neue Handymodelle ermöglichen jedoch polyphone Klingeltöne und farbige sowie animierte Betreiberlogos bzw. Bildschirmschoner.

\subsection{Forschungsfragen}

Angesichts der Popularisierung und ökonomischen Bedeutung von Klingeltönen und Logos ist es von Interesse, die sozialen und kommunikativen Bedeutungen dieser neuen Medien zu untersuchen. Klingeltöne und Logos auf dem Handy lassen sich insofern als neue Medien charakterisieren als sie a) Träger von Symbolen sind, b) auf einer bestimmten Übertragungstechnik basieren (Mobilfunk), c) ihre Produktion und Distribution in spezifischer Weise durch Medienunternehmen organisiert ist und sie d) gesellschaftlicher Aneignung und Sinngebung unterliegen, etwa durch kollektive Nutzungspraxen und öffentliche Diskurse. Die ersten bislang vorliegenden sozialwissenschaftlichen Sammelbände zur Handy-Kommunikation (Brown, Green \& Harper, 2001; Katz \& Aakhus, 2002a) befassen sich jedoch nicht näher mit der Klingelton- und Logo-Nutzung und einschlägige Zeitschriftenbeiträge fehlen bislang ebenfalls, weshalb die vorliegende Arbeit in Anlehnung an das Konstrukt der Uni-Kommunikation zunächst fünf grundlegende Forschungsfragen angeht:

- Wie häufig und mit welchen Methoden werden neue Klingeltöne und Logos auf das eigene Handy geladen (quantitativer Aspekt)?

- Wodurch wird die Häufigkeit von Klingelton- und Logo-Downloads determiniert?

- Welche Themen stehen bei Klingelton- und Logo-Downloads zur Verfügung und werden bevorzugt gewählt (qualitativer Aspekt)?

- Wodurch wird die Themenwahl bei Klingelton- und Logo-Downloads determiniert?

- In welchen sozialen Situationen werden Klingeltöne und Logos thematisch und wie reagieren Ohren- und Augenzeugen?

Das Konstrukt der Uni-Kommunikation hat bislang keine große Verbreitung erfahren, so dass weder eine elaborierte Theorie noch empirische Untersuchungsinstrumente zur Verfügung stehen. Dennoch liefert es hilfreiche Anregungen dazu, welche Determinanten und Konsequenzen bei der Klingelton- und Logo-Nutzung eine Rolle spielen könnten: So wird die Bedeutung der Identitätskonstruktion und Selbstdarstellung betont, woraus sich ergibt, dass zwischen den Selbstdarstellungs-Motiven des Individuums, seinen Interessen und Gruppenzugehörigkeiten (z. B. Altersgruppen, Geschlechtsgruppen) einerseits und der Klingelton- und Logo-Nutzung andererseits systematische Zusammenhänge bestehen müssten. Beim Vorliegen entsprechender Korrelationen wären Klingeltöne und Logos dann tatsächlich indikativ für die Merkmale der Zielperson. UniKommunikation und Selbstdarstellung implizieren Publikumsadressierung, wobei einerseits ein selektiertes Publikum gezielt mit einem Klingelton oder Logo konfrontiert werden kann (gezielte Selbstdarstellung), andererseits aber auch anonyme Publika (z. B. Passanten) ungezielt zu Ohren- oder Augenzeugen werden können (ungezielte Selbstdarstellung).

Uni-Kommunikation per Klingelton oder Logo funktioniert nur dann, wenn Außenstehende diese Medien überhaupt wahrnehmen und entsprechend reagieren - wobei sowohl innere (interpersonale Eindrucksbildung) als auch manchmal äußere (interperso- 
nale Kommunikation) Reaktionen zu erwarten sind. Folgt auf die öffentliche Präsentation von Klingelton und/oder Logo tatsächlich eine interpersonale Anschluss-Kommunikation, so müsste diese häufig als intra- oder intergruppale Kommunikation strukturiert sein: Indem nämlich Klingeltöne und Logos standardisierte Symbole der Populär-Kultur verwenden und Interessen oder Gruppenzugehörigkeiten spiegeln, legen sie es Personen nahe, sich nicht als singuläre Individuen zu erkennen und anzusprechen, sondern in erster Linie als Mitglieder der selben sozialen Gruppe (intragruppale Kommunikation; z. B. zwischen Fans derselben Musikrichtung, die einander anhand eines Handy-Klingeltons identifizieren) oder aber als Mitglieder einer Outgroup (intergruppale Kommunikation; z. B. zwischen Fans unterschiedlicher Musikrichtungen, die einander anhand eines Handy-Klingeltons identifizieren).

Da Klingeltöne und Logos als digitale Medien reproduzierbar sind, kann das Teilen einer Symbolwelt noch mit Interaktionen des Tauschens oder Schenkens gekoppelt werden und somit soziale Beziehungen stärken oder auch den eigenen sozialen Status bekräftigen, weil Anzahl und Art der Klingeltöne und Logos, über die eine Person als Ressourcen verfügt, sozialem Vergleich unterliegen.

Etablierte Forschungsfelder wie Massenkommunikation, politische Kommunikation oder Öffentlichkeitsarbeit anzuführen, um die vermeintliche Randständigkeit von UniKommunikation hervorzuheben, ist nicht sinnvoll, da Uni-Kommunikation typischerweise Botschaften aus den genannten Bereichen aufgreift, umwandelt und rekontextualisiert, wobei gerade der Aspekt der Gruppenzugehörigkeit von Bedeutung ist:

„The bumper sticker 'Jesus Saves', makes use of an automobile, which is both a means of transportation and a symbol, to carry an additional and more explicit message to fellow drivers. Such messages ordinarily do not originate with the person displaying them. Rather, they are mass produced and distributed by groups who are campaigning for certain causes. The persons displaying these messages become part of the campaign as well as part of the transmission system. This makes uni-communication different from other forms of interpersonal interaction. It communicates affiliation with a group or suggests a social role rather than making an individual statement. [...] Uni-communication discloses how the displayer views her or himself in affiliation with others rather than in relationship to an individual receiver." (Cathcart \& Gumpert, 1983: 276f.).

Das Konstrukt der Uni-Kommunikation weist also enge Verbindungen zu den sozialpsychologischen Konstrukten Selbstdarstellung (Leary, 1996), Eindrucksbildung (z. B. Jones, 1990, Kenny, 1994) sowie soziale Identifikation und intra- bzw. intergruppale Kommunikation (Tajfel, 1982; Tajfel \& Turner, 1979) auf, deren theoretische Modellierungen in zukünftigen Studien für die Elaboration eines uni-kommunikativen Modells fruchtbar gemacht werden könnten.

\section{Methoden}

Zur explorativen Beantwortung der fünf Forschungsfragen wurden drei empirische Studien durchgeführt: Eine Inhaltsanalyse von $n=10$ Websites mit Klingelton- und LogoAngeboten, eine leitfadengestützte mündliche Befragung von $\mathrm{n}=30$ Handy-Nutzern sowie eine vollstrukturierte Online-Befragung von $\mathrm{n}=808$ Handy-Nutzern. 


\subsection{Inhaltsanalyse}

Um die zur Verfügung stehenden Klingelton- und Logo-Angebote zu beschreiben wurden exemplarisch $\mathrm{n}=10$ deutschsprachige Websites mit entsprechendem DownloadBereich einer Inhaltsanalyse unterzogen. Die Anzahl der pro Website angebotenen einzelnen Klingeltöne und Logos belief sich jeweils auf mehrere Hundert, die auf durchschnittlich 17 Klingelton- und 34 Logo-Rubriken verteilt waren (siehe Tab. 1). Rubriken, unter denen die Anbieter jeweils thematisch ähnliche Klingeltöne und Logos gebündelt anbieten, lauten beispielsweise „Klassik“, „Pop“, „HipHop“, „Türkisches“, „Film \& Fernsehen“ (Klingeltöne) oder „Comics \& Cartoons“, „Sexy“, „Auto \& Verkehr“, „Sport \& Spiele“, „Biene Maja“ (Logos).

Tabelle 1: Klingelton- und Logo-Angebote auf 10 deutschsprachigen Websites

\begin{tabular}{|c|c|c|c|c|}
\hline $\begin{array}{l}\text { Websites mit } \\
\text { Klingelton- und } \\
\text { Logo-Angeboten }\end{array}$ & Unternehmen & $\begin{array}{l}\text { Reichweiten } \\
\text { der Websites } \\
\text { Stand: } 04 / 05.2002\end{array}$ & $\begin{array}{l}\text { Klingelton- } \\
\text { Rubriken }\end{array}$ & $\begin{array}{l}\text { Logo- } \\
\text { Rubriken }\end{array}$ \\
\hline $\begin{array}{l}\text { bild.t-online.de } \\
\text { (powered by jamba.de) }\end{array}$ & $\begin{array}{l}\text { Bild.T-online.de AG } \\
\text { \& Co. KG }\end{array}$ & 10.984.821 Visits (1) & 26 & 26 \\
\hline $\begin{array}{l}\text { bravo.de } \\
\text { (powered by handy.de) }\end{array}$ & $\begin{array}{l}\text { Heinrich Bauer Zeit- } \\
\text { schriften Verlag KG }\end{array}$ & 1.571.739 Visits (1) & 18 & 32 \\
\hline fun2handy.de & INA Germany AG & keine E-Mail-Antwort & 04 & 35 \\
\hline handy.de & $\begin{array}{l}\text { Handy.de Vertriebs } \\
\text { GmbH }\end{array}$ & $\begin{array}{l}\text { 1.043.804 Visits (1) } \\
\text { 2,8 Mio Kunden (2) }\end{array}$ & 18 & 40 \\
\hline handy-fantasy.de & $\begin{array}{l}\text { Logonaut Internet } \\
\text { Services GmbH } \\
\text { \& Co KG }\end{array}$ & 10.000 Visits (3) & 17 & 23 \\
\hline handystoff.de & $\mathrm{pmm} \mathrm{GmbH}$ & $\begin{array}{l}\text { keine Auskunft des } \\
\text { Anbieters }\end{array}$ & 19 & 83 \\
\hline jamba.de & Jamba! AG & $\begin{array}{l}\text { 8.000.000 Views (3) } \\
\text { 1,8 Mio Kunden (2) }\end{array}$ & 26 & 26 \\
\hline mobilemania.de & $\begin{array}{l}\text { Hot-Wire-Telekom.de } \\
\& \text { Human-Call.de }\end{array}$ & 180.000 Visits (3) & 17 & 24 \\
\hline $\begin{array}{l}\text { mtv.de } \\
\text { (powered by handy.de) }\end{array}$ & $\begin{array}{l}\text { MTV Networks } \\
\& \text { Co. OHG }\end{array}$ & 1.480.983 Visits (1) & 12 & 32 \\
\hline sms.de & [netzquadrat] $\mathrm{GmbH}$ & $\begin{array}{l}\text { keine E-Mail-Antwort } \\
\text { 0,5 Mio Kunden (2) }\end{array}$ & 11 & 22 \\
\hline Gesamt (Mittelwert) & & & 16,8 & 34,3 \\
\hline
\end{tabular}

(1) Visits laut IVW-Messung April 2002 www.ivw.de (2) registrierte Kunden laut Selbstauskunft des Anbieters auf der Website (3) Visits laut Selbstauskunft des Anbieters per E-Mail

Die Inhaltsanalyse anhand von zwei induktiv gewonnenen Kategoriensystemen bezog sich auf Anzahl und Art der inhaltlichen Rubriken für Klingeltöne und Betreiberlogos (siehe Kap. 3.3). 


\subsection{Leitfaden-Interviews}

Mit $\mathrm{n}=30$ Personen (15 weiblich, 15 männlich) im Alter von 13 bis 28 Jahren wurden Leitfaden-Interviews zur Nutzung von Klingeltönen und Handylogos durchgeführt. Die Befragungspersonen wurden teilweise an öffentlichen Orten (Fußgängerzone, Schnellrestaurant) ohne vorheriges Screening willkürlich rekrutiert und befragt, teilweise erfolgten auch Befragungen von Studierenden und ihren Bekannten. Gemäß Interview-Leitfaden wurde zunächst allgemein nach der Handy-Nutzung gefragt, dann speziell die Quantität und Qualität von Klingelton- und Logo-Downloads angesprochen, wobei insbesondere Gründe für die Auswahl bestimmter Klingelton-Melodien oder Logo-Motive sowie besondere Episoden oder Umstände dieser Form der UniKommunikation im sozialen Kontext zur Sprache kommen sollten. Nutzten die Befragten selbst keine Klingeltöne und Logos, so wurde dennoch nach Beobachtungen und Reaktionen auf entsprechende Uni-Kommunikation von anderen gefragt. Die Befragten zeigten sich dem Forschungsthema gegenüber aufgeschlossen und auskunftsbereit. Die Interviews wurden auf Tonband aufgezeichnet und für jedes Interview wurde ein Protokoll mit Nutzungsdaten und einschlägigen Zitaten angefertigt (siehe Kap. 3.5).

\subsection{Online-Befragung}

Ein standardisierter Online-Fragebogen zur Nutzung von Klingeltönen und Logos wurde entwickelt, an 40 Probanden vorgetestet und revidiert. In die Item-Konstruktion gingen Ergebnisse der Inhaltsanalyse und der Leitfaden-Interviews ein. Der Fragebogen bestand aus fünf thematischen Blöcken, wobei viele Variabeln durch Einzelfragen abgedeckt wurden, teilweise aber auch selbst konstruierte Kurzskalen zum Einsatz kamen (siehe Anhang):

1. Häufigkeiten der Klingelton- und Logo-Downloads und verwendete DownloadMethoden (z. B. „Wie oft haben Sie in den letzten zwei Monaten einen neuen Klingelton/ein neues Logo auf Ihr Handy geladen?")

2. Determinanten der Download-Häufigkeit: Nutzungshäufigkeit der verwandten $\mathrm{Me-}$ dien SMS und Bildmitteilung; Motivation zur Handy-Gestaltung; Geschlecht; Alter; Individualität im Sinne der Kultivierung eines eigenen Stils; Uniformität im Sinne einer Orientierung an aktuellen Trends; Klingelton- und Logo-Nutzung im sozialen Umfeld

3. Themenwahlen bei Klingelton- und Logo-Downloads (z. B. „Aus welchen der folgenden Rubriken haben Sie bereits Logos auf Ihr Handy geladen?")

4. Determinanten der Themenwahl (verschiedene Interessen, Geschlecht, Alter)

5. Sozialstatistik

Die Endfassung des Fragebogens enthielt 30 Items und beanspruchte etwa 10 bis $15 \mathrm{Mi}$ nuten Bearbeitungszeit, was für einen Online-Fragebogen relativ lang ist. Der OnlineFragebogen wurde von Dezember 2001 bis Januar 2002 im WWW veröffentlicht und durch Verlinkung von sieben Handy-Portalen sowie durch Postings in 25 Handy-Foren beworben. Zielgruppe des Fragebogens waren Personen, die sowohl an der Onlineals auch an der Mobilkommunikation teilnehmen und Klingeltöne und/oder Handylogos nutzen. Die Antworten wurden über ein Web-Formular eingelesen und die Daten mit dem Statistikprogramm SPSS ausgewertet. Der Datensatz enthielt kaum fehlende Werte und auch das überwiegend positive Feedback im Kommentarfeld des Fragebogens deutet darauf hin, dass der Fragebogen bei den freiwilligen Teilnehmern auf gute Akzeptanz stieß. 
Die auf diesem Wege gewonnene Selbstselektions-Stichprobe bestand aus $\mathrm{N}=808$ Personen (40\% weiblich, 60\% männlich) im Alter von 11 bis 55 Jahren. 38\% der Befragten waren im Alter von 11 bis 18 Jahren, $46 \%$ im Alter von 19 bis 28 Jahren und $16 \%$ im Alter von 29 bis 55 Jahren. Dementsprechend waren die finanziellen Mittel der Befragten eher gering: Zwei Drittel verfügten über weniger als 500 Euro pro Monat. Etwa die Hälfte der Befragten lebte in Städten bzw. Orten mit weniger als 20.000 Einwohnern, die andere Hälfte in größeren Städten. Die meisten Befragten besaßen ein Handy des Herstellers Nokia (75\%), dessen Endgeräte besonders vielfältige Möglichkeiten der Klingelton- und Logo-Nutzung zulassen. Diverse Download-Angebote sind auch nur auf Nokia-Handys zugeschnitten. Am zweithäufigsten vertreten waren Siemens-Handys (14\%). Zwei Drittel der Befragten besaßen ihr Handy seit mehr als zwei Jahren. Die Befragten gaben monatlich im Durchschnitt 38,90 Euro (Standardabweichung s $=48,88$ ) für ihre Handynutzung aus.

\section{Ergebnisse}

Aufgrund der willkürlichen Stichprobenziehungen haben die Ergebnisse der Mehrmethoden-Studie explorativen Charakter, das heißt, sie beanspruchen keine Repräsentativität für bundesdeutsche Handy-Nutzung. Sie liefern aber erste Anhaltspunkte zu den fünf Forschungsfragen nach Download-Häufigkeiten und Determinanten von Download-Häufigkeiten, nach Themen-Wahlen und Determinanten von Themen-Wahlen sowie nach sozialen Konsequenzen der Klingelton- und Logo-Nutzung in mehr oder minder öffentlichen Situationen.

\subsection{Häufigkeit von Klingelton- und Logo-Downloads}

Wie häufig werden neue Klingeltöne und Logos auf das eigene Handy heruntergeladen? Durchschnittlich 1,75 Mal pro Monat $(s=2,17)$ luden die Teilnehmerinnen und Teilnehmer der Online-Fragebogen-Studie einen neuen Klingelton und 1,71 Mal pro Monat $(s=2,11)$ ein neues Logo auf ihr Handy. Die Verteilungen der Download-Häufigkeiten von Klingeltönen und Logos unterschieden sich dabei nicht voneinander. Personen, die sich Klingeltöne herunterluden, nutzten in der Regel auch Logos $(r=.46$, $\mathrm{p}<.001)$. Ebenso gab es einen überzufälligen linearen Zusammenhang zwischen der Häufigkeit der Klingelton- und der Häufigkeit der Logo-Downloads ( $\mathrm{r}=.58, \mathrm{p}<.001$ ).

Hinsichtlich der Häufigkeit von neuen Klingelton- und Logo-Downloads ließ sich das Online-Sample in fünf Gruppen einteilen: 1. Tages-Nutzer (neuer Klingelton- oder Logo-Download nach wenigen Tagen bzw. häufiger als ein Mal pro Woche), 2. Wochen-Nutzer (Download nach ein, zwei oder drei Wochen), 3. Monats-Nutzer (Download nach ein oder zwei Monaten), 4. Jahres-Nutzer (neuer Klingelton- oder LogoDownload erst nach mehreren Monaten oder Jahren) und 5. Nicht-Nutzer (siehe Tab. 2).

Die verschiedenen Download-Methoden für Klingeltöne und Logos (vgl. Kap. 1.2) wurden von den Befragungspersonen unterschiedlich häufig in Anspruch genommen: Am häufigsten erfolgte der Download via Internet, also über eine Website, am seltensten wurde allein auf eine Service-Rufnummer zurückgegriffen (siehe Tab. 3). Hinsichtlich der Rangfolge der gewählten Download-Methoden zeigten sich keine Differenzen zwischen Klingeltönen und Logos. 
Tabelle 2: Fünf Nutzergruppen im nicht-repräsentativen Online-Sample gemäß Häufigkeit von neuen Klingelton- und Logo-Downloads $(N=808)$

\begin{tabular}{lcc}
\hline Download-Häufigkeit & Klingeltöne & Logos \\
\hline 1. Tages-Nutzer & $9 \%$ & $9 \%$ \\
2. Wochen-Nutzer & $26 \%$ & $25 \%$ \\
3. Monats-Nutzer & $29 \%$ & $34 \%$ \\
4. Jahres-Nutzer & $14 \%$ & $15 \%$ \\
5. Nicht-Nutzer & $22 \%$ & $17 \%$ \\
\hline
\end{tabular}

Tabelle 3: Häufigkeit (1: nie bis 4: oft) der Nutzung unterschiedlicher Download-Methoden im nicht-repräsentativen Online-Sample (arithmetische Mittelwerte, Standardabweichungen)

\begin{tabular}{lcc}
\hline Download-Methoden & Klingeltöne & Logos \\
\hline Websites & $2,98(1,01)$ & $3,11(0,97)$ \\
mitgeliefert auf dem Handy & $2,47(1,02)$ & $2,41(1,05)$ \\
Freunde & $2,07(1,01)$ & $2,04(0,95)$ \\
Editoren & $1,87(1,07)$ & $1,97(1,10)$ \\
Service-Rufnummern & $1,33(0,72)$ & $1,34(0,71)$ \\
\hline & $\mathrm{N}=629$ & $\mathrm{~N}=676$ \\
\hline
\end{tabular}

Ein quantitatives Maß für die Nutzungsintensität waren schließlich noch die monatlichen Kosten für Klingeltöne und Logos (siehe Tab. 4).

Tabelle 4: Logo und Klingelton-Nutzung und deren durchschnittliche monatliche Kosten in Euro $(M, s)$ im nicht-repräsentativen Online-Sample $(N=808)$

\begin{tabular}{lcc}
\hline & Logo-Nutzung & Keine Logo-Nutzung \\
\hline Klingelton-Nutzung & $72 \%(583)$ & $6 \%(46)$ \\
& $2,41(4,74)$ & $1,13(3,85)$ \\
Keine Klingelton-Nutzung & $11 \%(93)$ & $11 \%(86)$ \\
& $0,55(1,65)$ & $0,00(0,00)$ \\
\hline
\end{tabular}

Mit dem Online-Fragebogen zur Nutzung von Handy-Logos und Klingeltönen wurden durch Selbstselektion verstärkt Handybesitzerinnen und -besitzer rekrutiert, die besonderes Interesse an den fraglichen Medienprodukten hatten und diese überdurchschnittlich stark nutzten. So war die Gruppe der „Tages-Nutzer“ mit jeweils 70 Personen verhältnismäßig stark repräsentiert, allerdings befanden sich am anderen Ende des Spektrums auch Nicht-Nutzer im Sample. Auffallend ist die enorme Varianz der Download-Häufigkeiten bei den Nutzern: Personen, die einmalig einen Klingelton und ein Logo herunterladen und diese dann monate- oder jahrelang unverändert lassen, stehen Vielnutzern gegenüber, die regelmäßig nach einigen Tagen ihre Klingeltöne und Logos wechseln. Neben dieser interpersonalen Varianz sind jedoch noch intraindividuelle Veränderungen in der Download-Häufigkeit zu beachten, etwa wenn eine anfängliche Ex- 
perimentierphase nachlässt oder umgekehrt, wenn sich erst nach einer Weile regelmäßige Nutzungsmuster etablieren.

Da sozialwissenschaftliche Studien zur Klingelton- und Logo-Nutzung bislang fehlen, stehen Vergleichsdaten nur aus der Marktforschung zur Verfügung. Mit monatlichen Ausgaben von durchschnittlich 2,14 Euro $(s=4,46)$ für Klingeltöne und Logos erwiesen sich die hier befragten Handynutzer mit Internetzugang als besonders aktive Konsumenten auf dem Klingelton- und Logo-Markt, denn für norwegische HandyNutzer wurden die monatlichen Pro-Kopf-Ausgaben auf 0,45 Euro (5,43 Euro pro Jahr) beziffert (Strand Consult, 2001; vgl. Einleitung).

\subsection{Determinanten der Häufigkeit von Klingelton- und Logo-Downloads}

Häufige Klingelton- und Logo-Downloads sollten auf der Ebene des Umgangs mit der Technik mit einer verstärkten Nutzung ähnlicher Medien (SMS, Bildmitteilungen) sowie mit einer Motivation zur Handy-Gestaltung einhergehen. Letztere beinhaltet das von Joachim Höflich und Patrick Rössler (2001) bereits im Zusammenhang mit SMSNutzung identifizierte Motiv, „die technischen Möglichkeiten des Geräts auszuprobieren" (zur Operationalisierung siehe Anhang). Tatsächlich zeigten sich entsprechende positive Zusammenhänge (siehe Tab. 5), die hier als Determinanten plausibilisiert werden, wobei Kausalitätsnachweise weiteren Studien vorbehalten sind.

Auf Seiten des Individuums zeigte sich kein Alterseffekt. Ein Geschlechtseffekt wurde in der Weise deutlich, dass sich unter den Vielnutzern (bzw. Tagesnutzern, siehe oben Tab. 2) etwa doppelt so viele Jungen und Männer wie Mädchen und Frauen befanden. Andererseits zeigte eine Umfrage von $\mathrm{N}=2.979$ norwegischen Schülern, dass 50\% der Mädchen und 43\% der Jungen Klingeltöne „sehr wichtig“ fanden (Skog, 2002), so dass Geschlechtseffekten noch genauer nachzugehen wäre. Selbststilisierungen im Sinne von Individualität („Ich habe meinen eigenen Stil.“; siehe Anhang) sowie von Uniformität („Wenn etwas gerade im Trend ist, mache ich gerne mit.“; siehe Anhang) korrelierten positiv mit Klingelton- und Logo-Downloads, wobei sich Logo-Downloads durch diese Variablen besser vorhersagen ließen. Individualität und Uniformität im hier definierten Sinne stehen insofern nicht in Widerspruch zueinander, als man sich mit häufig wechselnden Klingeltönen und Logos zwar von anderen Nutzern abhebt, gleichzeitig die Distinktionssymbole aber aus dem allgemein zugänglichen Pool populärkultureller Symbole stammen (sofern nicht Eigenproduktionen vorgenommen werden).

Technikaneignung unterliegt gerade bei Netzmedien starker sozialer Normierung (vgl. Fulk, Schmitz \& Steinfield, 1990). Und tatsächlich erwiesen sich Nutzungsintensität und Nutzungsnormen im sozialen Umfeld („Viele meiner Freunde nutzen sehr häufig Klingeltöne oder Logos.“; „Meine Freunde finden Klingeltöne und Logos gut.“, siehe Anhang) als der beste Prädiktor für die individuellen Download-Häufigkeiten sowohl im Sinne der bivariaten Korrelationen als auch der Beta-Gewichte im Kontext der multiplen Regression.

Trotz der verwendeten Kurzskalen und ihrer teilweise suboptimalen internen Konsistenzen (siehe Anhang) konnten durch die multiple Regression substanzielle Varianzanteile aufgeklärt werden (19\% bei den Klingeltönen, $16 \%$ bei den Logos), die sich durch eine verbesserte Operationalisierung vermutlich noch steigern ließen. Wer einen individuellen Stil oder eine allgemeine Trendorientierung kultiviert, lädt etwas häufiger Klingeltöne und vor allem Logos auf das eigene Handy, was ein Indiz für deren unikommunikative Aneignung ist. Ein Reiz von Klingeltönen und Logos scheint in der Handy-Gestaltung zu liegen, hier wäre der Frage nachzugehen, inwiefern damit eine 
uni-kommunikative Selbstbestätigung und Imagepflege hinsichtlich der eigenen Technikkompetenz verbunden ist. Die Nutzungsintensität von Klingeltönen und Logos als digitalen (und damit kopierbaren und selbst distribuierbaren) Netzmedien korreliert eng - und vermutlich enger als bei vielen anderen Medien der Uni-Kommunikation wie z. B. bedruckten T-Shirts - mit der Nutzungsintensität im sozialen Umfeld.

Tabelle 5: Determinanten der Häufigkeit von Klingelton-und Logo-Downloads ( $r$ : bivariate Korrelationen; $b$ : nur signifikante beta-Koeffizienten in der multiplen Regression $R ; *: p<.05$ )

\begin{tabular}{|c|c|c|c|c|}
\hline Prädiktoren & $\begin{array}{l}\mathbf{r} \\
\text { Klingelton- } \\
\text { Downloads }\end{array}$ & $\begin{array}{l}\mathrm{b} \\
\text { Klingelton- } \\
\text { Downloads }\end{array}$ & $\begin{array}{l}\mathbf{r} \\
\text { Logo- } \\
\text { Downloads }\end{array}$ & $\begin{array}{l}\mathrm{b} \\
\text { Logo- } \\
\text { Downloads }\end{array}$ \\
\hline \multicolumn{5}{|l|}{ Technik } \\
\hline 1. SMS & .08 & & $.13 *$ & \\
\hline 2. Bildmitteilungen & $.29 *$ & $.16^{*}$ & $.24 *$ & $.15^{*}$ \\
\hline 3. Skala Handy-Gestaltung & $.28 *$ & $.16^{*}$ & $.24 *$ & $.13 *$ \\
\hline \multicolumn{5}{|l|}{ Individuum } \\
\hline 4. Geschlecht (1:w, 2:m) & .07 & $.11 *$ & $.13 *$ & $.19 *$ \\
\hline 5. Alter & -.05 & & .01 & \\
\hline 6. Skala Individualität & .04 & & $.11 *$ & \\
\hline 7. Skala Uniformität & $.16 *$ & & $.10 *$ & \\
\hline \multirow[t]{2}{*}{$\begin{array}{l}\text { Soziales Umfeld } \\
\text { 8. Skala Klingelton- } \\
\text { bzw. Logo-Nutzung }\end{array}$} & $.37 *$ & $.25 *$ & $.32 \%$ & $.22 \%$ \\
\hline & $\mathrm{N}=600$ & $\begin{array}{l}\mathrm{R}=.44, \\
\mathrm{p}<.001 \\
\mathrm{R}^{2} \text { korr }=19 \%\end{array}$ & $\mathrm{~N}=640$ & $\begin{array}{l}\mathrm{R}=.41, \\
\mathrm{p}<.001 \\
\mathrm{R}^{2} \text { korr }=16 \%\end{array}$ \\
\hline
\end{tabular}

Die Interview-Studie zeigte, dass Nicht-Nutzer vor allem drei Gründe für ihre Klingelton- und Logo-Abstinenz nannten: 1. Ihr Handytyp erlaubte keine zusätzlichen Klingeltöne oder Logos, 2. entsprechende Angebote wurden als zu teuer eingestuft oder 3. nicht für sinnvoll befunden („Das interessiert mich einfach nicht. Hauptsache, das Handy klingelt“; „Hat nichts mit dem Preis zu tun, das Handy ist kein Spielzeug für mich“).

\subsection{Themenwabl bei Klingelton- und Logo-Downloads}

Welche Themen stehen für Klingelton- und Logo-Downloads zur Verfügung und werden bevorzugt gewählt? Bei den Klingeltönen und Logos, die auf das Handy geladen werden, handelt es sich entweder um vorproduzierten mobilen Content oder um Eigenproduktionen. Das Angebot an vorproduziertem Content ist entsprechenden Download-Sites im WWW oder auch Zeitschriften-Anzeigen zu entnehmen. Unter dem Aspekt der Uni-Kommunikation interessiert vor allem, welche selbstbezogenen Aussagen durch die Wahl von Klingeltönen und Logos getroffen werden können.

Eine Annäherung an das Aussagen-Potenzial der Klingeltöne und Logos erfolgte durch eine Inhaltsanalyse der Klingelton- und Logo-Rubriken auf 10 ausgewählten Websites. Das Kategoriensystem wurde - mangels einer theoretisch ableitbaren, trenn- 
scharfen und erschöpfenden Taxonomie von identitätsrelevanten Themen - sowohl für Klingeltöne als auch für Logos induktiv gewonnen (siehe Tab. 6 und Tab. 7). Hierzu wurden die vorgefundenen Rubriken in der Weise gebündelt und etikettiert, dass ihre Aussagekraft für individuelle Präferenzen oder Gruppenzugehörigkeiten deutlich wird. Klingelton-Rubriken wie „Pop“, „Rock“, „Klassik“ oder „Charts“ lassen etwa Rückschlüsse auf den Musikgeschmack einer Person zu. So berichteten Jugendliche in den Leitfaden-Interviews, sie würden sich gezielt ihr „Lieblingslied“ als Klingelton herunterladen oder ihren jeweiligen Favoriten aus der aktuellen Hitparade. Manche monierten auch, dass Klingelton-Anbieter den von ihnen bevorzugten Musikstil (z. B. „Techno") in ihrem Angebot nicht genügend berücksichtigen. Die uni-kommunikative Bedeutung von Klingeltönen, die bestimmte Musikstile oder auch einzelne Interpreten repräsentieren, besteht prinzipiell darin, dass eine Person ihre Musikorientierung durch die Wahl des Klingeltons unterstreichen und dem Umfeld unausgesprochen mitteilen kann, so dass Anhänger derselben Stilrichtung einander ebenso erkennen (intragruppale Kommunikation), wie Fans unterschiedlicher Musikstile sich voneinander abheben (intergruppale Kommunikation).

Die Zuordnung der auf den Websites vorgefundenen Rubriken zu den thematischen Kategorien der Inhaltsanalyse erfolgte durch zwei unabhängige Kodierer, wobei über alle 168 Klingelton-Rubriken hinweg eine sehr gute Inter-Coder-Reliabilität zu beobachten war (ICR $=.98$, Cohens Kappa).

Die Auswertung zeigte, dass bei den Klingeltönen der Schwerpunkt tatsächlich auf Musikstilen liegt, die sehr ausdifferenziert darstellbar sind: $61 \%$ der Klingelton-Rubriken der $\mathrm{n}=10$ betrachteten Websites beinhalteten Musikstile. Sie fehlten auf keiner einzigen der 10 Websites und umfassten Klassik und Country ebenso wie Volksmusik, Neue Deutsche Welle, Pop, Rock, HipHop oder die aktuellen Charts.

Mit vielfältigen Konnotationen versehen sind Erkennungsmelodien von Fernseh-Serien sowie Filmmusiken: Von „Dallas“ über die „Sendung mit der Maus“ bis zu „Miss Marple“, „Ally McBeal“, „Star Trek“, „James Bond“ oder „Indiana Jones“ reicht das Spektrum, wobei trotz monophonischer Realisation (vgl. Kap. 1.2) teilweise eine verblüffend leichte Wiedererkennbarkeit der Melodien gegeben ist, die interessanterweise häufig gerade durch eine Veränderung der Original-Melodie z. B. hinsichtlich Tonart, Tonumfang, Notenlänge erreicht wird.

Während Klingeltöne aus den Rubriken „Musik“ und „Film/TV“ vor allem Rückschlüsse auf den Geschmack des Nutzers zulassen und Fangemeinschaften als soziale Bezugsgruppen salient machen, lassen Rubriken wie „Nationalhymnen“ oder „Türkisches“ Bezüge zur Nationalität und Rubriken wie „Kinderlieder“ oder „Oldies“ Bezüge zur Generation anklingen. Der aus dem Konstrukt der Uni-Kommunikation abgeleitete Ansatz, inhaltliche Klingelton- und Logo-Rubriken mit Selbst-Aspekten bzw. Identitäten zu verknüpfen, ist hier freilich nur als erste Annäherung zu verstehen. Doch es scheint zunächst plausibel, dass mit türkischen Klingelton-Melodien vermutlich eine entsprechende nationale Identität unterstrichen wird. Und es hat sich gezeigt, dass ältere Nutzer tatsächlich verstärkt „Oldies“ herunterladen, so dass dieser Rubrik ein Generations-Thema zugeschrieben werden kann (vgl. Kap. 3.4). Schließlich sind eine Reihe spezifischer Interessen und Vorlieben durch entsprechende Klingeltöne darstellbar: Produkt- bzw. Firmen-Werbung etwa für Coca-Cola, Haribo oder MacDonalds, Fußball-Hymnen, Sounds von Computerspielen und Jagdsignale. Weniger identitätsbezogen sind dagegen saisonale Klingeltöne (v. a. Weihnachtslieder) sowie abstrakte Handysounds, die in Klang, Form und Darbietungsart so nur mit dem Handy realisierbar sind. 
Es ist unter ökonomischen Gesichtspunkten davon auszugehen, dass KlingeltonThemen, die von Anbietern besonders umfassend abgedeckt werden, gleichzeitig auch jene sind, die sich bei den Nutzern größerer Beliebtheit erfreuen. So produziert etwa Jamba.de jede Woche gezielt rund fünf neue Klingeltöne und zwanzig neue Logos für die laut Webstatistik am häufigsten nachgefragten Downlaod-Rubriken. Tatsächlich zeigte sich, dass die meisten befragten Klingelton-Nutzer (87\%) im Online-Sample schon mindestens einmal einen Klingelton aus der Kategorie „Musik“ heruntergeladen hatten (siehe Tab. 6). Klingeltöne mit Bezügen zu Nation oder Generation wurden von einer Minderheit von gut 10\% genutzt, auf Erkennungsmelodien von Fernsehserien oder Filmmusiken hatte dagegen schon fast jede/r zweite zurückgegriffen.

Tabelle 6: Klingelton-Themen: Angebot und Nutzung

\begin{tabular}{|c|c|c|c|c|}
\hline $\begin{array}{l}\text { Kategorien von } \\
\text { Klingelton-Themen } \\
\text { (alphabetisch) }\end{array}$ & $\begin{array}{l}\text { Beispiele für } \\
\text { Klingelton-Rubriken } \\
\text { laut Anbieter }\end{array}$ & $\begin{array}{l}\text { Anteil der } \\
\text { Websites } \\
(\mathrm{N}=10 \\
\text { Websites })\end{array}$ & $\begin{array}{l}\text { Anzahl der } \\
\text { Rubriken } \\
(\mathrm{N}=168 \\
\text { Rubriken })\end{array}$ & $\begin{array}{l}\text { Wahl der } \\
\text { Themen } \\
(\mathrm{N}=629 \\
\text { Klingelton- } \\
\text { Nutzer }) \\
\end{array}$ \\
\hline 1. Computer & Computerspiele & $20 \%$ & 2 & \\
\hline 2. Feiertage & Weihnachtslieder & $30 \%$ & 3 & \\
\hline 3. Film/TV & Film/TV & $90 \%$ & $11(6 \%)$ & $45 \%$ \\
\hline 4. Fußball & $\begin{array}{l}\text { Fan-Hymnen, } \\
\text { Fußball WM } 2002\end{array}$ & $60 \%$ & 8 & \\
\hline 5. Generation & Oldies, Kinderlieder & $70 \%$ & $10(6 \%)$ & $13 \%$ \\
\hline 6. Handysounds & $\begin{array}{l}\text { Handysounds, } \\
\text { Motorola Töne }\end{array}$ & $80 \%$ & & \\
\hline 7. Jagd & Jagdsignale & $10 \%$ & 1 & \\
\hline 8. Musik & Pop, Rock, Klassik, Charts & $100 \%$ & $103(61 \%)$ & $87 \%$ \\
\hline 9. Nation & $\begin{array}{l}\text { Türkisches, National- } \\
\text { hymnen }\end{array}$ & $90 \%$ & $13(8 \%)$ & $11 \%$ \\
\hline 10. Produkte/Firmen & Werbung, Jingles & $80 \%$ & 8 & \\
\hline 11. Sonstiges & Sonstiges & $10 \%$ & 1 & \\
\hline
\end{tabular}

Aus Platzgründen konnten im Online-Fragebogen nicht alle thematischen Kategorien abgefragt werden, so dass Angaben zu Nutzungshäufigkeiten nur für ausgewählte Themen vorliegen.

Ebenso wie die Klingelton-Rubriken wurden dann auch die Logo-Rubriken der 10 Websites in der Weise thematisch gebündelt, dass auf selbstbezogene Aussagen geschlossen werden kann (Inter-Coder-Reliabilität des Kategoriensystems über alle 343 Logo-Rubriken: ICR = .92). Es zeigte sich, dass Logo-Angebote qualitativ ein sehr viel breiteres Spektrum an Themen abdecken als Klingelton-Angebote: Von den elf Klingelton-Themen (siehe oben Tab. 6) waren 8 auch als Logo-Themen vertreten $(2,5,8,10$, 15, 16, 18, 23; Generation und Jagd fehlten als Themen, ebenso die akustisch abstrakten "Handysounds", mit denen jedoch die grafisch abstrakten Logo-"Muster" korrespondieren, siehe Tab. 7). Zusätzlich zu den Themen des Klingelton-Kategoriensystems wurden zwölf weitere Themen-Kategorien aufgenommen. 
Das breitere Themenspektrum auf Seiten der Medienangebote spiegelte sich in einem stärker aufgefächerten Nutzungsverhalten wider, wobei „Cartoons“ (53\%), „Muster“ (38\%), „Liebe“ (35\%) und „Sprüche“ (28\%), die jeweils die grafische und verbale Qualität des Mediums Logo ausnutzen, besonders beliebt waren.

Tabelle 7: Logo-Themen: Angebot und Nutzung

\begin{tabular}{|c|c|c|c|c|}
\hline $\begin{array}{l}\text { Kategorien von } \\
\text { Logo-Themen } \\
\text { (alphabetisch) }\end{array}$ & $\begin{array}{l}\text { Beispiele für } \\
\text { Logo-Rubriken } \\
\text { laut Anbieter }\end{array}$ & $\begin{array}{l}\text { Anteil der } \\
\text { Websites } \\
(\mathrm{N}=10 \\
\text { Websites })\end{array}$ & $\begin{array}{l}\text { Anzahl der } \\
\text { Rubriken } \\
\text { (N=343 } \\
\text { Rubriken) }\end{array}$ & $\begin{array}{l}\text { Wahl der } \\
\text { Themen } \\
(\mathrm{N}=676 \\
\text { Logo- } \\
\text { Nutzer) }\end{array}$ \\
\hline 1. Auto/Motorrad & Auto, Motorrad, Fahrzeuge & $90 \%$ & 16 & $09 \%$ \\
\hline 2. Berufe & Berufe, Business & $70 \%$ & 8 & \\
\hline 3. Cartoons & $\begin{array}{l}\text { Cartoons, Comics, } \\
\text { Lustiges, Fun }\end{array}$ & $100 \%$ & $28(8 \%)$ & $53 \%$ \\
\hline 4. Computer & $\begin{array}{l}\text { Computer, Technik, } \\
\text { Handy }\end{array}$ & $60 \%$ & 9 & \\
\hline 5. Feiertage & $\begin{array}{l}\text { Weihnachten, Ostern, } \\
\text { Muttertag }\end{array}$ & $70 \%$ & 16 & \\
\hline 6. Film/TV & Film/TV & $80 \%$ & $27(8 \%)$ & $17 \%$ \\
\hline 7. Fußball & $\begin{array}{l}\text { Fan-Hymnen, } \\
\text { Fußball WM } 2002\end{array}$ & $70 \%$ & 8 & \\
\hline 8. Liebe & Liebe & $100 \%$ & 10 & $35 \%$ \\
\hline 9. Musik & Musik & $90 \%$ & 14 & $14 \%$ \\
\hline 10. Muster & $\begin{array}{l}\text { Muster, Symbole, } \\
\text { Ornamente }\end{array}$ & $90 \%$ & $21(6 \%)$ & $38 \%$ \\
\hline 11. Mystery/Horror & Mystery, Horror, Gruseliges & s $90 \%$ & 9 & $17 \%$ \\
\hline 12. Namen & Namen, Vornamen & $40 \%$ & 8 & $27 \%$ \\
\hline 13. Nation & Türkisches, Asiatisches & $70 \%$ & 9 & \\
\hline 14. Produkte/Firmen & Firmen, Marken & $70 \%$ & 12 & \\
\hline 15. Reisen/Länder & Reisen, Länder, Urlaub & $80 \%$ & 10 & $07 \%$ \\
\hline 16. Sex & Sex, Erotik, Sexy, Hotgirls & $100 \%$ & 13 & $17 \%$ \\
\hline 17. Sport/Spiele & Sport, Spiele & $100 \%$ & 12 & \\
\hline 18. Sprüche & Sprüche, Bekenntnisse & $100 \%$ & $19(6 \%)$ & $28 \%$ \\
\hline 19. Sternzeichen & Sternzeichen & $100 \%$ & 10 & \\
\hline 20. Tiere/Natur & Tiere, Natur, Pflanzen & $100 \%$ & 15 & $27 \%$ \\
\hline 21. Sonstiges & $\begin{array}{l}\text { Sonstiges, Top Download, } \\
\text { Weltraum, Freibeuter/Pira- } \\
\text { ten, Esoterik, Drugs }\end{array}$ & $100 \%$ & 69 & \\
\hline
\end{tabular}

Aus Platzgründen konnten im Online-Fragebogen nicht alle thematischen Kategorien abgefragt werden, so dass Angaben zu Nutzungshäufigkeiten nur für ausgewählte Themen vorliegen. 
Durch die enge Verknüpfung mit dem Musikmarkt spielen bei den Klingelton-Angeboten urheber- und lizenzrechtliche Fragen eine größere Rolle als bei den Logo-Angeboten, wo sie primär in der Rubrik „Cartoons“ oder „Produkte/Firmen“ virulent werden. Der Anbieter Handy-Fantasy.de hatte zum Untersuchungszeitpunkt etwa sein Klingelton-Angebot vorläufig eingestellt:

\begin{abstract}
„Liebe Kunden von HANDY-FANTASY,
da zur Zeit fast alle Musikverlage sowie diverse Einzelinterpreten alle Anbieter von Klingeltönen abmahnen und teils horrende Summen fordern, können wir leider innerhalb der nächsten Wochen keine Klingeltöne mehr von unserer Homepage direkt versenden, solange bis die Rechtslage eindeutig geklärt ist. Traurig ist insbesondere, dass wir hohe GEMA-Gebühren gezahlt haben, wovon die Konzerne und Interpreten zwar auch profitiert haben, uns aber jetzt trotzdem abmahnen. Anstatt sich zu freuen, dass Ihr mit Euren Klingeltönen für Eure Idole gratis Werbung macht, wird uns verboten, diese Melodien für Euch bereitzustellen. Kaum verständlich, oder?“ (www.handyfantasy.de; 28. Mai 2002)
\end{abstract}

Während es bei der Produktion von Klingeltönen um das Nachkomponieren bekannter Melodien geht und Neukompositionen kaum gefragt sind, können im Bereich der Logos originelle Neukreationen wirkungsvoll sein. Somit beschäftigen die Logo-Anbieter teilweise eigene Designer, die fortlaufend neue Motive entwerfen. Manchmal wird auch den Nutzern die Möglichkeit geboten, ihre Eigenkreationen auf dem Server zur Verfügung zu stellen. Bei Handy.de werden die User-Logos nach dem selben Rubriken-Schema eingeteilt wie die vom Anbieter produzierten Logos.

Dass bei den Handylogos ein individualisierendes Moment stärker zum Tragen kommt, zeigt sich nicht nur in der verglichen mit Klingeltönen größeren Vielfalt der Themen, sondern auch darin, dass Logos tatsächlich etwas häufiger als Klingeltöne mit Editoren selbst erstellt werden (siehe oben Tab. 3) und dass die Logo-Nutzung auch überzufällig mit der Individualitäts-Skala korreliert (siehe oben Tab. 5). So berichtete ein 23-jähriger Auszubildender aus der Computerbranche im Interview, er würde niemals auf vorgefertigte Logo-Motive zurückgreifen, sondern immer mit Editoren arbeiten, schließlich „geht's ja um das Individuelle“. Wer per Editor kein eigenes Design entwerfen kann oder will, mag als individuelle Lösung immer noch eigene Fotovorlagen in Logos umwandeln lassen.

\title{
3.4 Determinanten der Themenwabl von Klingelton- und Logo-Downloads
}

Das Konstrukt der Uni-Kommunikation besagt, dass Menschen Klingeltöne und Logos in der Weise auswählen, dass darin ihre Interessen und Gruppenzugehörigkeiten zum Ausdruck kommen. Dementsprechend müsste sich etwa die Identifikation mit einem bestimmten Musikstil (z. B. „Pop“ oder „Rock“) auch in einer überzufällig häufigeren Wahl von Klingeltönen aus dieser Rubrik niederschlagen. Die stärkere Affinität von Jungen und Männern zu „Film/TV“ müsste sich im Vergleich zu Frauen in einer häufigeren Nutzung diesbezüglicher Klingeltöne (und Logos) ebenso niederschlagen wie ältere Menschen häufiger als Jüngere Oldies als Klingeltöne wählen sollten.

Bei der thematischen Klingelton-Wabl war der Effekt des Musikgeschmacks durchweg sehr deutlich: Diejenigen, die besonderes Interesse an Musik aus den Charts angaben, hatten mit überwältigender Mehrheit bereits mindestens einmal einen Klingelton aus dieser Rubrik gewählt (85\%), während von den Klingelton-Nutzern ohne ausdrückli- 
ches Interesse an den Charts nur 31\% einen Klingelton aus dieser Rubrik genutzt hatten. Thomas, Fan der schwedischen Band „Millencolin“, berichtete im Interview, dass er bevorzugt T-Shirts mit Motiven und Tourdaten seiner Lieblingsband trägt und sich für sein neues Handy auch gleich das Millencolin-Logo von der Band-Homepage heruntergeladen hat. Er nutzt also ergänzend verschiedene Medien der Uni-Kommunikation, die von der Band bereitgestellt werden. Geschlechtseffekte zeigten sich nicht nur in der Rubrik „Film/TV“, sondern auch bei einzelnen Musikrichtungen. Der systematische Alterseffekt, der für das Thema „Generation“ (Rubrik „Oldies“) und für die Rubrik „Klassik“ nachweisbar war, zeigte sich komplementär für „HipHop“ - einem Musikstil, der offensichtlich Jugendkultur repräsentiert.

Um die selbstdarstellerische bzw. identitätsbezogene Bedeutung von Klingeltönen genauer zu untersuchen, wären weitere qualitative Befragungen sinnvoll, die individuelle Interpretationen des Medienangebots enthüllen. So werden einzelne Musikstücke teilweise nicht ausgewählt, weil sie einen bevorzugten Musikstil repräsentieren, sondern weil sie den Nutzer an eine bestimmte Situation erinnern oder weil der Liedtext sie anspricht. Der 25-jährige Danny berichtete im Interview, er hätte sich „Der kleine Trompeter" als Klingelton auf sein Handy geladen - weniger, weil er Arbeiterkampflieder mag, sondern eher, weil er selbst Gitarre und Trompete spielt. Ein Student aus den alten Bundesländern berichtete, er verwende die Nationalhymne der DDR als Klingelton, „weil ich in Ilmenau studiere und es einfach eine ausgeglichen komponierte Melodie ist".

Bei der Wahl der Handylogos, die inhaltlich ein viel weiteres Spektrum an SelbstAspekten adressieren können, zeigte sich erwartungskonform ein sehr deutlicher Einfluss aller thematisch korrespondierenden Interessen bzw. Hobbys (siehe Tab. 9). Stereotypkonforme Geschlechtseffekte traten prägnant zu Tage, etwa in der Weise, dass das Thema „Auto/Motorrad“ kaum von weiblichen (3\%), dagegen nennenswert von männlichen $(14 \%)$ Personen in der Stichprobe gewählt wurde. Weibliche Personen wählten doppelt so oft Liebes-Motive wie männliche, umgekehrt wählten männliche Personen doppelt so oft Sex-Motive wie weibliche. Dennoch standen Liebes-Motive mit 24\% auch bei Jungen relativ hoch im Kurs und wurden Sex-Motive von den befragten Mädchen (10\%) nicht durchgängig verschmäht. Alters- bzw. Generationseffekte zeigten sich bei diversen Themen, wobei die Attraktivität der jeweiligen Themen typischerweise mit dem Alter abnahm, bestenfalls gleich blieb. Lediglich bei dem Thema „Reisen/Länder" war ein Anstieg der Themenwahl mit dem Alter zu beobachten, was darauf hindeutet, dass die angebotenen Logo-Themen vor allem auf die jüngere Generation zugeschnitten sind.

Die in der Online-Befragung nachgewiesene enge Verbindung zwischen Themenwahlen einerseits und Interessen, Geschlechtszugehörigkeit und Altersgruppe andererseits belegt das uni-kommunikative Potenzial von Klingeltönen und Logos, das auch die Interview-Partner bestätigten: So berichtete ein 23-jähriger Angestellter, er habe sich als Logo ein „Schwert“ heruntergeladen, das sei „schlicht und geradlinig“ und war das einzige Logo, das ihm gefallen habe und seine Persönlichkeit widerspiegele. Seine Motivwahl korrespondiert mit der Beobachtung einer Schülerin, die im Interview meinte, „Jungs haben brutale Logos, Messer und so“, während bei Mädchen eher „Teddys und Herzen“ auf dem Handydisplay zu sehen seien. Ein 22-jähriger Student, dessen Hobby Fantasy-Rollenspiele sind, wählte deswegen ein keltisches Symbol als Handy-Logo. Ein 13-jähriger Realschüler meinte, dass er Klingeltöne nach „Lust und Laune“ auswählt, Logos dagegen „nach seiner Meinung, zum Beispiel ,Nazis raus““.

In den Interviews gab es auch Hinweise auf Zusammenhänge zwischen Themenwahl und Download-Häufigkeit. Nutzer, die ihre Klingeltöne und Logos häufiger - etwa bin- 


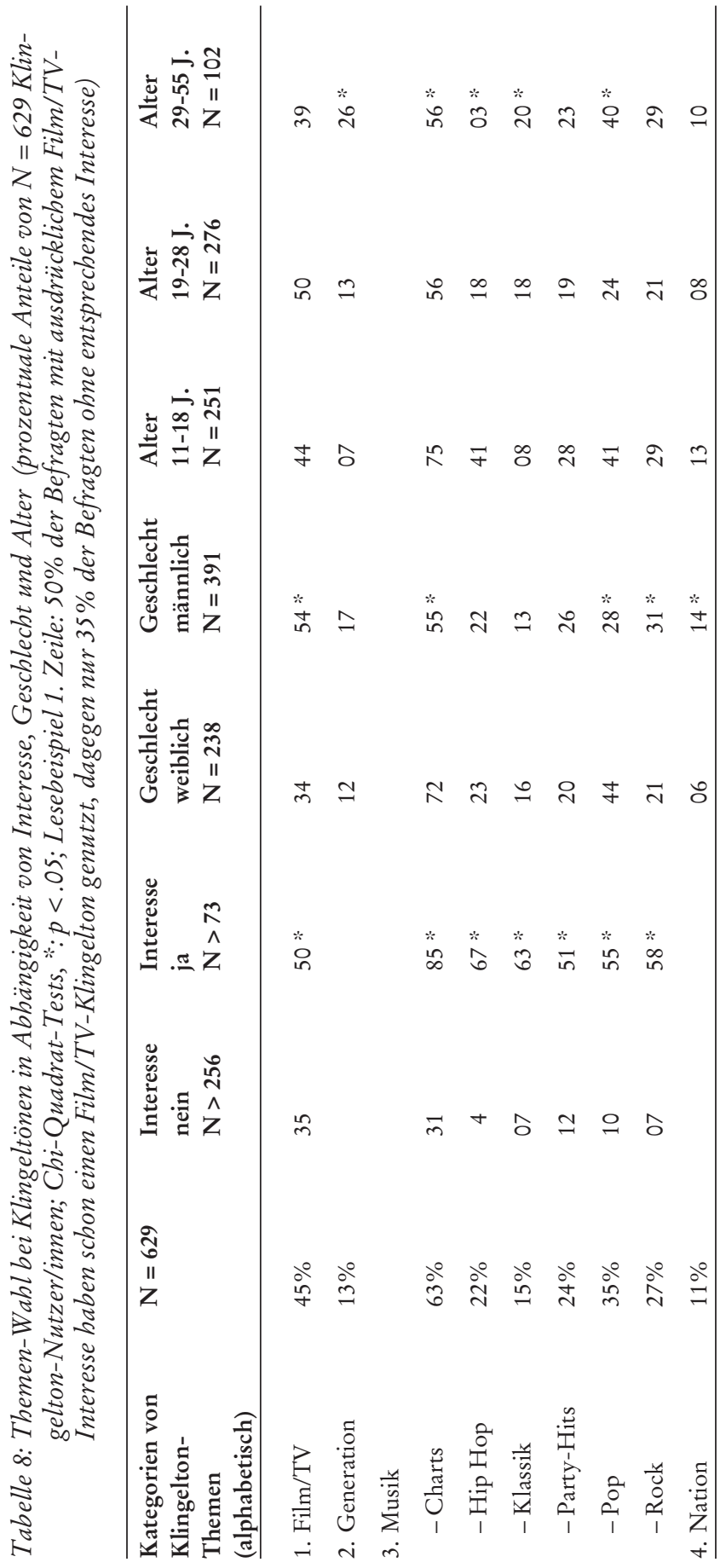




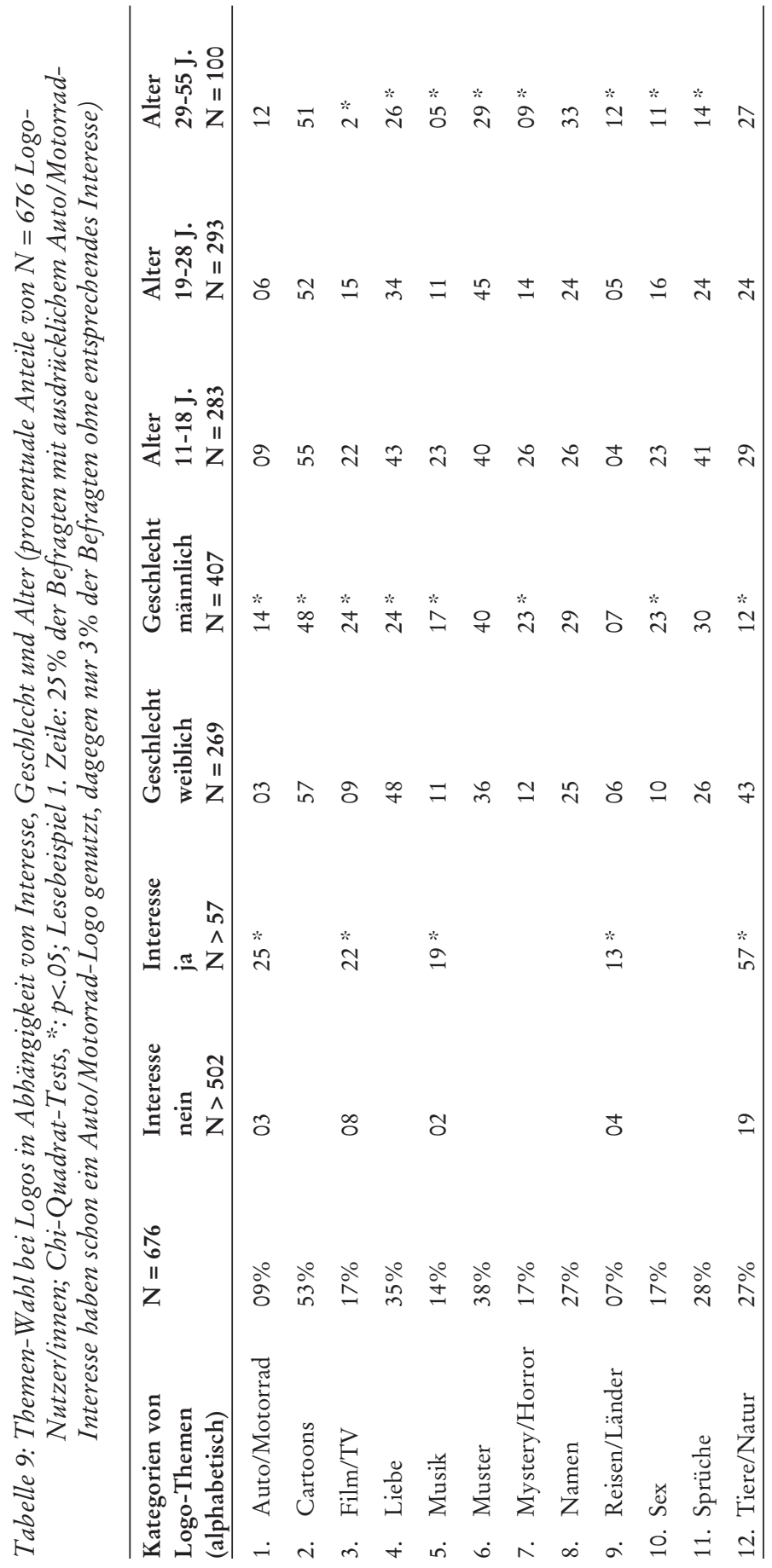


nen einiger Wochen - verändern, greifen eher auf Motive zurück, die per se eine turnusmäßige Aktualisierung nahe legen (z. B. Klingeltöne aus der Rubrik „Charts“ oder Logos aus der Themenkategorie „Feiertage“). Viel-Nutzer, die bereits nach einigen Tagen ihre Klingeltöne und/oder Logos austauschen, sind innerhalb der von ihnen präferierten Themenfelder offensichtlich von originellen oder trendigen Neuerscheinungen fasziniert. Ein 28-jähriger studentischer Vielnutzer berichtete, er sei „immer auf der Suche nach witzigen Logos. Das ist eine Sammlerleidenschaft wie bei mp3“.

\subsection{Soziale Konsequenzen der Klingelton- und Logo-Nutzung}

Im Rahmen der Leitfaden-Interviews wurden insgesamt zehn ausführliche Episoden zu situationsspezifischen sozialen Konsequenzen der Klingelton- und Logo-Nutzung erzählt. Diese Situationen (ergänzt um zwei Online-Quellen) behandeln gezielte und ungezielte Selbstdarstellungen samt Reaktionen sowie intra- und intergruppale AnschlussKommunikation.

\section{Gezielte Selbstdarstellung}

Bei der gezielten Selbstdarstellung verwendet der Handybesitzer Klingeltöne und vor allem Logos bewusst, um sich gegenüber den gerade Anwesenden in Szene zu setzen: Aktiv spielt er anderen seinen Klingelton vor oder präsentiert das Display seines Handys, was Alexandra Weilenmann und Catrine Larsson (2002: 95f.) auf der Basis ihrer Feldbeobachtungen im Zusammenhang mit SMS-Kommunikation als „minimal forms of sharing" bezeichnen, das heißt, nicht das Handy wird verliehen („physical sharing“), sondern der symbolische Inhalt wird sozial geteilt. Dieses kann beim Publikum auf positive Resonanz, auf Gleichgültigkeit oder auch auf Ablehnung stoßen, wie die folgenden fünf Episoden illustrieren:

- Bei einer Party führte Hannes, ein Auszubildender, seinen Kumpels sein neuestes Logo aus der Rubrik Cartoons vor: Es zeigte neben dem Schriftzug „BSE“ eine „verrückte Kuh“. Das Logo wurde allgemein als witzig eingestuft. Sein Besitzer war eine ganze Weile Zentrum der Aufmerksamkeit und reüssierte als Unterhalter. Er zeigte sein Handy herum und verschickte das Logo spontan an einige Interessierte, die Logo-fähige Handys bei sich hatten.

- Der 28-jährige Markus legte bei einem Restaurantbesuch mit Freunden sein Handy auf den Tisch, so dass die anderen sein Logo aus der Rubrik Produkte/Firmen prompt bemerkten und nachfragten. Es handelte sich um das Logo der Firma, bei der er seit Neuestem beschäftigt ist. Erfreut über das Interesse an seiner Arbeit und seiner Firma berichtete er ausführlich und war ganz in seinem Element.

- Sichtlich stolz war Julia, eine Psychologiestudentin, die ihren neuesten Klingelton aus der Rubrik Film/TV („Miss Marple“) zwei Freunden ausgiebig vorspielte. Diese konnten sich jedoch wenig für den Klingelton erwärmen. Die Ironie ihrer „Ganz toll!“-Ausrufe bemerkte die enthusiastische Handybesitzerin nach Auskunft des einen Freundes jedoch nicht.

- Juliane, eine 13-jährige Schülerin, nutzt ein Logo aus der Rubrik Namen („Jule“), das ihr ein Verwandter aus dem Internet besorgte. Eine Mitschülerin kommentiert: „Sie will sich eben immer abheben. Sie wird auch immer ganz zickig, wenn man mal das gleiche Büchlein oder Hausaufgabenheft hat wie sie. Aber mit dem Handy will sie sich vielmehr in der Klasse beliebt machen, sie wird nämlich nicht so besonders anerkannt. Da hat sie doch tatsächlich zu uns gesagt, wir dürfen ihr Handy mitbenut- 
zen. Das Logo soll auf jeden Fall immer jeder sehen, da tut sie so, als ob sie sich das Handy ansieht oder SMS schreibt oder spielt - nur damit niemand in der Klasse es übersieht.“

- Eine ähnlich offensive, gezielte Selbstdarstellung wie Juliane betreibt auch die 13jährige Sandra gegenüber ihren Mitschülern, nachdem sie von ihren Eltern das allerneueste Motorola-Handy geschenkt bekommen hat, das neben herkömmlichen Betreiberlogos auch Bildschirmschoner unterstützt. Eine Mitschülerin kommentiert: "Sandra hat als Bildschirmschoner Batman, der in seinem Auto an einer Mauer vorbeifährt. Woher ich das weiß? Natürlich, weil sie es einem förmlich unter die Nase hält. Neulich wurde sie in Mathe an die Tafel gerufen und hat tatsächlich in ihrer scheinbar extra dafür vorgesehenen Gürteltasche das Handy stecken gehabt, so dass es auch um jeden Preis die ganze Klasse sieht." Selbst bei Jugendlichen kann die gezielte Präsentation des Handys offenbar schnell übertrieben selbstdarstellerisch wirken und auf Ablehnung stoßen. Die Kunst der Selbstdarstellung besteht schließlich darin, sie möglichst beiläufig zu bewerkstelligen.

\section{Ungezielte Selbstdarstellung}

Situationen, in denen Logos und vor allem Klingeltöne spontan, ohne Ausrichtung auf konkrete Adressaten und vielleicht sogar ungewollt die Aufmerksamkeit von Ohrenund Augenzeugen auf sich ziehen, werden hier im Unterschied zur oben beschriebenen gezielten Selbstdarstellung als „ungezielt“ etikettiert.

- Ein Angestellter aus England berichtet, wie peinlich es ihm war, ausgerechnet zu einem wichtigen Geschäfts-Meeting zu spät zu kommen. Kaum saß er am Konferenztisch, begann auch noch sein Handy zu klingeln, das er in der Eile nicht ausgeschaltet oder lautlos gestellt hatte. Was die Situation rettete, war jedoch sein sofort als Jim'll Fix erkennbarer Klingelton, der für allgemeine Erheiterung und sogar ausgelassenes Mitsingen sorgte. So nachzulesen in einer auf der Homepage des KlingeltonProduzenten Phat Tones (www.tonez.co.uk) publizierten Kunden-E-Mail. (Demgegenüber betonte ein älterer Informant im Interview, es wäre ihm schon unangenehm genug, wenn das Handy in der Öffentlichkeit klingelt, ein besonders auffälliger Klingelton wäre da „umso peinlicher“.)

- Wie sich der Klingelton in der Öffentlichkeit als eine erfolgreiche Aufmerksamkeitsressource erweisen kann, erzählt eine Studentin: „Wir saßen zu dritt in einem Café auf dem Ku'damm und plötzlich ging ein Handy los und es erklang die Melodie von Samba de Janeiro, diesem Sommerhit von vor 2 Jahren und man erkannte das auch relativ schnell und gut. Das Handy gehörte einer jungen attraktiven dunkelhaarigen Frau, die sehr sommerlich - kurzer Rock, Top - bekleidet war. Sofort wurden ein paar junge Männer am Nachbartisch aufmerksam und grinsten sich an. Einer von denen sagte dann sowas wie: ,Die sieht schon so aus, als hätte sie richtig Feuer im Hintern.' 'Genau konnte ich das nicht verstehen, aber es war sowas in der Art. Schließlich konnte ich dann noch sehen, wie einer der Männer die Frau, nachdem sie fertig war mit telefonieren, anlächelte und sie mit ihm flirtete."

\section{Intragruppale Kommunikation}

Intragruppale Kommunikation findet statt in Situationen, in denen Mitglieder derselben sozialen Gruppe einander anhand von Klingeltönen oder Logos erkennen bzw. bewusst ihren Gruppenzusammenhalt stärken. 
- Rolf wurde von einem Bekannten auf sein Handylogo der TV-Serie The Simpsons angesprochen. Beide kamen überein, dass die Serie ziemlich „cool“ sei und freuten sich, ineinander Gleichgesinnte gefunden zu haben. Danach folgte die Frage, ob man die letzte Folge gesehen habe und das Gespräch ging über in Nacherzählungen einzelner besonders lustiger Episoden aus der Folge vom Vorabend.

- Das Irish Pub „Carnaby“ in Gotha kommuniziert seinen Kneipennamen samt SofaMotiv über die Leuchtreklame, die Speisekarte, eine Anstecknadel und neuerdings auch über ein Handylogo. Nicht nur der Inhaber und die Angestellten, sondern auch die ersten Stammgäste nutzen das Logo aus der Rubrik Produkte/Firmen. In dem Pub, in dem es recht familiär zugeht, ist das neue Logo oft Gesprächsthema, etwa weil weitere Gäste es sich herunterladen wollen.

- Eine Engländerin berichtet, dass sie und ihre Bürokolleginnen sowohl gerne Schokolade essen, als auch wechselseitig ihre Klingeltöne beachten. Momentan nutzt sie einen Klingelton aus der Rubrik Produkte/Firmen und schlägt dem Klingelton-Produzenten Phat Tonez in einer auf dessen Website publizierten E-Mail eine Neuproduktion vor: „I am also a big chocoholic, so the Cadburys Fruit and Nut advert is currently my favourite ringtone. Can I suggest though that you try to make a ringtone of the music from the old Ferrero Rocher advert? All my friends at work would be mad with jealousy if I had this on my phone as we all love those chocolates."

- Die Mitarbeiter einer Multimedia-Firma, die gemeinsam an einem Projekt für den Automobilhersteller Audi arbeiteten, trafen zu Projektbeginn spontan die Entscheidung, sich alle ein Audi-Logo auf ihre Handys zu laden, wodurch sie ihren Zusammenhalt als Team stärkten und sich von den anderen Mitarbeitern der Firma abhoben.

\section{Intergruppale Kommunikation}

Intergruppale Kommunikation findet statt in Situationen, in denen Mitglieder unterschiedlicher sozialer Gruppen einander anhand von Klingeltönen und Logos erkennen und sich infolgedessen voneinander abgrenzen.

- $\quad$ Seit kurzem zeigt das Handy der 21-jährigen Farnoush ein Logo aus der Rubrik Musik: „3p - mehr bass“ ist das Symbol des Frankfurter Musik-Labels 3P „Pelham Power Productions“. Sie mag deren Rap-Musik. Auf das Logo wird sie immer wieder angesprochen. Weil viele diese Rap-Musik nicht gut finden, sind das meistens negative Âußerungen wie „was, du hörst solche Musik?“. Das ist für Farnoush ein „kalkulierbares Risiko“, denn sie wird wegen ihres Musikgeschmacks oft „dumm angemacht“. Dass überhaupt ein entsprechendes Logo zur Verfügung steht, verdankt sie der offensiven Merchandising-Politik der Plattenfirma. Moses Pelham selbst berichtet im Interview (Seifert, 2000): „Wir haben [...] schon T-Shirts im Copy-Shop gemacht, als wir noch keine einzige Platte hatten. Und heute sind wir die einzigen deutschen Rapper, die es schaffen ein T-Shirt mit so einem abstrakten Logo wie „3p-mehr bass“ vorne drauf zu verkaufen.“

\section{Diskussion}

Handy-Klingeltöne und Handy-Logos sind der bislang ökonomisch wichtigste mobile Content. Dabei geht es zum einen um den direkten Vertrieb dieser digitalen Produkte, zum anderen aber auch um neue crossmediale Marketing-Strategien etwa in der Musikoder Comicbranche. Indem Handy-Nutzerinnen und -Nutzer ihre Endgeräte mit Klin- 
geltönen und Logos ausstatten, in denen sich ihre Interessen, Geschlechts- und Altersrollen oder andere Gruppenzugehörigkeiten widerspiegeln, eignen sie sich ihr Endgerät gestalterisch an, bekräftigen sie ihre Identität, pflegen sie soziale Beziehungen und strukturieren interpersonale Kommunikation als intra- oder intergruppale Begegnung mit entsprechender Anschluss-Kommunikation. Gleichzeitig sorgen sie auch dafür, dass Symbole der glokalisierten Populärkultur jenseits der Massenmedien-Rezeption in die Lebenswelten integriert werden: Da erklingt dann der Sound von „Dallas“ auf dem Schulhof oder sieht man das BWM-Logo auf dem Handy des Fahrgastes im Bus.

Eine kommunikationswissenschaftliche Zuwendung zu dem hier in seinen vielfältigen Fassetten vorgestellten neuen Gegenstandsgebiet der Klingeltöne und Logos scheint lohnenswert, wobei sich das Konstrukt der Uni-Kommunikation als fruchtbar erwiesen hat. Zwar mögen Klingeltöne und Logos auf Seiten der Nutzer teilweise ohne kommunikative Absicht verwendet und/oder von Außenstehenden als Botschaften ignoriert werden. Doch die vorliegende Pilotstudie belegt, dass viele Nutzer Klingeltöne und Logos offensichtlich in Übereinstimmung mit identitätsbezogenen Kategorien auswählen, dass sie sie gezielt anderen Menschen zeigen und dass Ohren- und Augenzeugen wiederum diese Botschaften beachten und auf sie reagieren. Insgesamt ist das bislang wenig rezipierte Konstrukt der Uni-Kommunikation elaborierungsbedürftig, sowohl was die theoretischen Bezüge zu verwandten Konstrukten betrifft (z. B. Selbstdarstellung, soziale Identifikation) als auch seine Operationalisierung.

Auch wäre der bereits 1975 von Gary Gumpert geäußerten These nachzugehen, dass Uni-Kommunikation zunehmend an Bedeutung gewinnt. Dies scheint plausibel, wenn man in Rechnung stellt, dass durch Urbanisierung, Globalisierung und wachsende Mobilität Individuen häufiger vor anonymen Publika auftreten und beobachtbare Zeichen der sozialen Zugehörigkeit eine willkommene soziale Orientierungshilfe bieten dürften. Auch die Online-Kommunikation, bei der sich Individuen mit persönlichen Homepages an unterschiedliche (oft anonyme) Publika wenden und dabei zum Teil auf vorproduzierten Content zurückgreifen (Döring, 2001), ist im Zusammenhang mit UniKommunikation zu sehen. Der Trend zur maßgeschneiderten Massenfertigung von Gebrauchsgegenständen (mass customization) und zu selbst gestaltbaren technischen Geräten, die körpernah getragen werden und den Charakter von Accessoires annehmen, vermehrt die Möglichkeiten zur Uni-Kommunikation. Vielversprechend scheint dabei nicht zuletzt eine genauere thematische Analyse und Kontrastierung von Objekten und Symbolen, die zur Uni-Kommunikation zur Verfügung stehen, und von Selbst-Aspekten, deren öffentliche Präsentation für Menschen relevant ist.

\section{Anhang}

Im Online-Fragebogen wurden vier Kurzskalen verwendet, um Determinanten der Häufigkeit von Klingelton- und Logo-Downloads zu operationalisieren. Die Skalen wurden über Summenscores ausgewertet, wobei alle selbstbeschreibenden Aussagen auf einer vierstufigen Ratingskala (stimmt gar nicht - wenig - ziemlich - völlig) zu beantworten waren. Die forschungsökonomisch notwendige Skalenkürze schlägt sich in teilweise geringen internen Konsistenzwerten (Cronbachs Alpha) nieder.

\section{Technik: Skala Handy-Gestaltung}

Klingelton $\alpha=.69 ; \operatorname{Logo} \alpha=.64$

Ein Klingelton [Logo] ist für mich wichtig, weil... 
1. ... ich mein Handy damit selbst gestalten kann.

2. ... ich $\mathrm{Spaß} \mathrm{daran} \mathrm{habe,} \mathrm{etwas} \mathrm{Neues} \mathrm{mit} \mathrm{meinem} \mathrm{Handy} \mathrm{auszuprobieren.}$

3. ... es praktisch zur Erkennung meines Handys ist.

4. ... es sich schön anhört [es schön aussieht].

5. ... es eine nette Spielerei ist.

6. ... ich finde, dass es eine nützlich technische Option des Handys ist.

\section{Individuum: Skala Individualität}

$\alpha=.50$

1. Ich lege großen Wert darauf, mich von der Masse abzuheben (z. B. durch Mode, Musik, Handy).

2. Ich habe meinen eigenen Stil (z. B. bei Mode, Musik, Handy).

\section{Individuum: Skala Uniformität}

$\alpha=.52$

1. Wenn etwas gerade im Trend ist, mache ich gerne mit (z. B. bei Mode, Musik, Handy).

2. Ich habe in vielen Bereichen den gleichen Geschmack wie meine Freunde (z. B. bei Mode, Musik, Handy).

\section{Soziales Umfeld: Skala Klingelton- und Logo-Nutzung}

$\alpha=.83$

1. Viele meiner Freunde nutzen sehr häufig Klingeltöne oder Logos.

2. Klingeltöne und Logos sind häufig Gesprächsthema in meinem Freundeskreis.

3. Ich tausche oft Klingeltöne und Logos mit meinen Freunden.

4. Meine Freunde finden Klingeltöne und Logos gut.

\section{Literatur}

Brown, Barry, Green, Nicola \& Harper, Richard (Eds.) (2001). Wireless World. Social and Interactional Aspects of the Mobile Age. London: Springer.

Cathcart, Robert \& Gumpert, Gary (1983). Mediated Interpersonal Communication. Toward A New Typology. Quarterly Journal Of Speech, 69 (3), 267 - 277.

Döring, Nicola (2001). Persönliche Homepages im WWW. Ein kritischer Überblick über den Forschungsstand. Medien E Kommunikationswissenschaft, 49 (3), 325 - 349.

Döring, Nicola (2002).,,1x Brot, Wurst, 5Sack Äpfel I.L.D.“ - Kommunikative Funktionen von Kurzmitteilungen (SMS). Zeitschrift für Medienpsychologie 3/2002.

Fortunati, Leopoldina (1997). The ambigious image of the mobile phone. In Leslie Haddon (Ed.), Communication on the move - The Experience of Mobile Telephony in the 1990's. http://web.cnlab.ch/cost248/repository/Experience_Mobile's/ communications_on_the_move_.html [6.6.2002]

Fulk, Janet, Schmitz, Joseph \& Steinfield, Charles W. (1990). A Social Influence Model of Technology Use. In Janet Fulk \& Charles Steinfield (Eds.), Organizations and Communication Technology (pp. 117-140). Newbury Park: Sage.

GEMA (Gesellschaft für musikalische Aufführungs- und mechanische Vervielfältigungsrechte) (2001). GEMA Brief 39 - Nene GEMA-Tarife für den Online-Bereich. http://www.gema.de/ kommunikation/briefe/ [6.6.2002]

Gumpert, Gary (1970). The rise of mini-comm. Journal of Communication, 20, $280-290$. 
Gumpert, Gary (1975). The rise of uni-comm. Today's Speech, Fall 1975, $34-38$.

Gumpert, Gary \& Drucker, Susan (1999). Models, Metaphors, and Other Scholarly Meanderings: Renovating Models of Communication. The New Jersey Journal of Communication, 7 (1), 9-22.

Harper, Richard (2002). The Mobile Interface: Old Technologies and New Arguments. In Barry Brown, Nicola Green \& Richard Harper (Eds.), Wireless World. Social and Interactional Aspects of the Mobile Age (pp. 207 - 226). London: Springer.

Höflich, Joachim R. (2001). Das Handy als „persönliches Medium“. Zur Aneignung des Short Message Service (SMS) durch Jugendliche.kommunikation@gesellschaft, 2 (1). http://www.kommunikation-gesellschaft.de/ [6.6.2002]

Höflich, Joachim R. \& Rössler, Patrick (2001). Mobile schriftliche Kommunikation - oder: E-Mail für das Handy. Die Bedeutung elektronischer Kurznachrichten (Short Message Service) am Beispiel jugendlicher Handynutzer. Medien E Kommunikationswissenschaft 49 (1), 437 - 461.

International Telecommunication Union (2002). World Telecommunication Development Report 2002: Reinventing Telecoms. http://www.itu.int/ITU-D/ict/publications/wtdr_02/[6.6.2002]

Jones, Edward E. (1990). Interpersonal Perception. New York: Freeman.

Jupiter MMXI (2002). Paid Content More Successful on Mobile than on PC. Press Release $17 \mathrm{Ja}-$ nuary 2002. http://uk.jupitermmxi.com/xp/uk/press/releases/pr_011702.xml [6.6.2002]

Katz, James E. \& Aakhus, Mark A. (Eds.) (2002a). Perpetual Contacts. Mobile Communication, Private Talk, Public Performance. Cambridge: Cambridge University Press.

Katz, James E. \& Aakhus, Mark A. (2002b). Introduction: Framing the issues. In James E. Katz \& Mark A. Aakhus (Eds.), Perpetual Contacts. Mobile Communication, Private Talk, Public Performance (pp. 1 - 14). Cambridge: Cambridge University Press.

Kenny, David (1994). Interpersonal Perception. A Social Relations Analysis. New York: Guilford Press.

Leary, Mark R. (1996). Self-Presentation: Impression Management and Interpersonal Bebavior. New York: Westview Press.

Lycett, John E. \& Dunbar, Robin I. M. (2000). Mobile Phones as Lekking Devices among Human Males. Human Nature 11 (1), 93 - 104.

Plant, Sadie (2001). On the mobile. The effects of mobile telephone on social and individual life. http://www.motorola.com/mot/documents/0,1028,297,00.doc [6.6.2002]

Robbins, Katheleen A. \& Turner, Martha A. (2002). United States: popular, pragmatic and problematic. In James E. Katz \& Mark A. Aakhus (Eds.), Perpetual Contacts. Mobile Communication, Private Talk, Public Performance (pp. 80-93). Cambridge: Cambridge University Press.

Seifert, Sascha (2000). Moses P. - Das Kind was rappt. Moses Pelham und 3P - Mehr Bass! Interview im September 1998. Trotzlust - Eine Magazinrevue für Menschen mit Sinn für eigene Sichtweisen, 23.3.2000. http://www.trotzlust.de/archiv/1999_2001/archiv/interview/991115_ moses\%20pelham [6.6.2002]

Skog, Berit (2002). Mobiles and the Norwegian teen: identity, gender and class. In James E. Katz $\&$ Mark A. Aakhus (Eds.), Perpetual Contacts. Mobile Communication, Private Talk, Public Performance (pp. 255 - 273). Cambridge: Cambridge University Press.

Strand Consult (2001). SMS - The Key to a billion dollar market. Strand Report. http://www. strandreports.com/sw69.asp [6.6.2002]

Tajfel, Henry (1982). Social psychology of intergroup relations. Annual Review of Psychology, 33, $1-30$.

Tajfel, Henry \& Turner, John C. (1979). An integrative theory of intergroup conflict. In William G. Austin \& Stephen Worchel (Eds.), The social psychology of intergroup relations (pp. 33 - 47). Monterey, CA: Brooks/Cole.

Townsend, Anthony M. (2002). Communications in the Twenty-first Century City. In Barry Brown, Nicola Green \& Richard Harper (Eds.), Wireless World. Social and Interactional Aspects of the Mobile Age (pp. 62 - 78). London: Springer.

Weilenmann, Alexandra \& Larsson, Catrine (2002). Local Use and Sharing of Mobile Phones. In Barry Brown, Nicola Green \& Richard Harper (Eds.), Wireless World. Social and Interactional Aspects of the Mobile Age (pp. 92-107). London: Springer.

Zykla, Jenni (2001). Schicken ist föhn. Sex T-Shirt-Sprüche sind modern. die tageszeitung, 26.6.2001. http://www.taz.de/pt/2001/06/26/a0122.nf/text [6.6.2002] 